\title{
A Rotational Motion Perception Neural Network Base on Asymmetric Spatiotemporal Visual Information Processing
}

\author{
Bin $\mathrm{Hu}$, Shigang Yue, Member, IEEE, Zhuhong Zhang
}

\begin{abstract}
All complex motion patterns can be decomposed into several elements, including translation, expansion/contraction and rotational motion. In biological vision systems, scientists have found specific types of visual neurons have specific preferences to each of the three motion elements. There are computational models on translation and expansion/contraction perception, however, little has been done in the past to create computational models for rotation motion perception. To fill this gap, we proposed a neural network which utilizes a specific spatiotemporal arrangement of asymmetric lateral inhibited directional selective neural networks for rotational motion perception. The proposed neural network consists of two parts presynaptic and postsynaptic parts. In the presynaptic part, there are a number of lateral inhibited directional selective neural networks to extract directional visual cues. In the postsynaptic part, similar to the arrangement of the directional columns in the cerebral cortex, these directional selective neurons are arranged in a cyclic order to perceive rotational motion cues. In the postsynaptic network, the delayed excitation from each directional selective neuron is multiplied by the gathered excitation from this neuron and its unilateral counterparts depending on which rotation, clockwise or counterclockwise, to perceive. Systematic experiments under various conditions and settings have been carried out and validated the robustness and reliability of the proposed neural network in detecting clockwise or counterclockwise rotational motion. This research is a critical step further towards dynamic visual information processing.
\end{abstract}

Index Terms - Rotational Selective Neuron, Directional Columns, Asymmetric Lateral Inhibition, Directional Selective Neurons, Multiplication, Visual Motion Perception, Spatiotemporal Computation

Manuscript revised and received on 14.05.2016, accepted on 29.05.2016 This work is partially supported by EU FP7 Project HAZCEPT (318907), LIVCODE (295151), Horizon 2020 project STEP2DYNA (691154), and National Natural Science Foundation of China (Grant No: 61563009) (Corresponding author: Shigang Yue).

B. Hu is with the College of Computer Science and Technology, Guizhou University, Guiyang, 550025, China, and also with the School of Computer Science, University of Lincoln, Lincoln LN6 7TS, UK (e-mail: csuhubin@163.com).

S. Yue is with the School of Computer Science, University of Lincoln, Lincoln LN6 7TS, UK (e-mail: syue@lincoln.ac.uk; yue.shigang@ieee.org; yue.lincoln@gmail.com)

Z. Zhang is with the College of Big Data and Information Engineering, Guizhou University, Guiyang, 550025, China (e-mail: sci.zhzhang@gzu.edu.cn).

Digital Object Identifier

\section{INTRODUCTION}

$\mathrm{F}^{\mathrm{O}}$ OR most animals, visual perception plays the most important role for their survival. With efficient visual perception, animal actively perceives and captures useful visual information about its external environments, such as an object's motion, shape, color and so on, and converges them to the higher cerebral cortex for decision making. Among these visual information perceptions, motion perception is particularly important for the survival of most animal species in critical moments, such as, to detect predators or to hunt for prey.

In the real world, the diversity of motion patterns can be decomposed into three types of basic motion elements or patterns, including translation, expansion/contraction and rotational motion [1], [2]. These basic motion patterns play important roles in motion synthesis, for instance, a moving wheel contains translation and rotational motion.

In biological vision systems, scientists have found specific types of visual neurons have specific preferences to each of the three motion elements. For example, Hubel and Wiesel discovered simple, complex and hyper-complex neuron types demonstrate orientation and velocity sensitivity characteristics [3]. In visual neurophysiological studies, three types of neurons have been found in the dorsal part of medial superior temporal (MSTd), ventral intraparietal area (VIP), anterior region of the superior temporal polysensory area (STPa) and area 7a in primates' brain [4]-[18] - these neurons are translation, rotation and expansion/contraction neurons which respond preferentially to translation, rotation, or expansion/contraction motion patterns.

For rotational selective neurons in biological vision systems, a number of studies have found its presence in the cerebral cortex of primate. For example, Leinonen reported neurons in Posterior Area 7 responding positively to rotational stimuli in the awake monkey [19]. Rizzolatti found similar neurons in the premotor cerebral cortex [20]. Sakata et al. found that rotation-sensitive neurons in the posterior parietal association cerebral cortex (area PG) of the alert monkey [5], [6]. Saito et al. found that some neurons in medial superior temporal (MST) area of the anesthetized monkey were sensitive to rotary movement [21]. Tanaka and Saito analyzed the functional properties of the rotational selective neurons with the direction, expansion/contraction neurons of MSTd in more detail, and proposed that a circular arrangement of movement directions in the wide-field stimuli was essential for the activation of rotation 
neurons [8]. Duffy and Wurtz also found that some neurons in the MSTd are sensitive to circular motion of optic flow stimuli, and further discover that the MSTd neurons respond not only to translation, expansion/contraction, or rotation, but often to two or all three of these motions [10], [11]. Rotation selective neurons have also been found in other animal species, such as human and birds. Cavanagh et al. found the human visual system contains the rotation detectors by demonstrating rotational motion aftereffects [22]-[26]. Koban and Nankoo found the pigeons are most sensitive to rotational motion in their research [27], [28]. All the above research suggests the existence of the rotational selective neurons in the biological visual pathways. However, the underlying mechanism of how a biological vision system perceives rotational motion remains unclear. There is few appropriate computational models focus on rotational motion perception in the past, let alone systematic investigation on the performance of such a rotational selective neural model.

On the other hand, constructing a rotational selective neural network based on the updated information revealed in neurophysiological studies may not only provide a building block for future artificial vision systems, but also help to further understand biological vision systems.

In this paper, we propose a bio-plausible rotational motion perception visual neural network based on the latest discoveries in neurophysiological studies. It makes use of asymmetrical lateral inhibited presynaptic neural networks and spatiotemporal circular arrangements as postsynaptic structure to achieve rotational motion perception. The proposed neural network models, for clockwise and counter clockwise respectively, are validated with systematic tests under various conditions.

The rest of the paper is organized as follows: in section II, related work will be reviewed; in section III, the proposed neural networks are described in detail; in section IV, experiments are carried out to test the performance of the new neural network system in different conditions.

\section{RELATED WORK}

In this section, we describe the related work from qualitative models in neurophysiology study, approaches with classical neural networks, quantitative models, to the studies link rotational to directional selective neurons.

Up to now, motion perceptions of translation and expansion/contraction have been modeled and tested (e.g. [29]-[33]). But few quantitative rotational perception models have been proposed, alongside several classical neural networks models as detailed below. To our knowledge, there is no bio-plausible quantitative model for rotational motion perception in literature up to date.

\section{A. Qualitative models in neurophysiology study}

In the study of cerebral neurophysiology, Saito et al. proposed a neural network which makes use of partially overlapping compartments in an MST receptive field [21]. This model needs a special surround effect in middle temporal (MT) neurons to prevent many compartments from being activated simultaneously. In Duffy's model, the local rotation and expansion of the velocity field is derived and summed up across space to get invariant responses [11]. This algorithm requires that MT neurons be selective to local rotation and expansion/contraction, which is generally not the case [7]. Orban et al. combined physiological recording and modeling techniques, proposed a computational model by using Gaussian-shaped tuning function [34]. However, the value of the standard deviation in the Gaussian-shaped tuning function affect the position invariance feature of this model; larger standard deviation value will make the model increasingly selective to the location of the center of rotation.

\section{B. Quantitative models for rotational motion perception}

In quantitative models, King et al. designed a neural network consists of multi-layered velocity sensitive sensory cells organized in a locally connected fashion [35], however the neural network cannot distinguish the direction of the rotational motion (a clockwise or a counterclockwise). Guo et al. proposed a model for the perception of rotational motion base on the Reichardt's correlation motion detector array, the Kohonen's self-organized feature map and the Schuster-wagner's oscillating neural network [36], how these neural networks are trained and the performance of the network is not clear.

The above two models demonstrated periodic responses to rotation motion with different structures - the functionality similar to our presynaptic part which can be excited in a sequential way to a rotational motion. Without postsynaptic part for specific rotational motion perception, the above two models are hard to compare with our model due to their incompleteness.

\section{Classical neural network approaches}

With different classical neural networks and learning rules, many other researchers have also proposed approaches to detect rotational motion, such as [37]-[42]. However, these classical neural networks need to learn from or train with a large number of rotational motion samples in order to work properly. Like other classical neural networks, their robustness and underlying biological origin are questionable.

\section{Research links directional to rotational selective neurons}

In the neurophysiological study of rotational motion perception, Sakata et al. reported the functional properties of rotation selective neurons in the posterior parietal association cerebral cortex of monkey [6], [8], [43], [44]. Sakata et al. found the fact that a pair of spots moving around the fixation point is as effective as a solid bar rotating in their experiment. This phenomenon suggests that the changing motion direction is more important than the changing object orientation. Therefore Sakata et al. made a point that the continuous change of motion direction in rotational motion is the only difference that distinguishes rotary from linear movement [6], [43], which is supported by Caplovitz and Tse's study on retinotopic area V3A in human [45]. This suggests that a rotational perception model could be created if it can detect the continuous changes of the motion direction of an object. These instantaneous 
motion directions of an object could be sensed by directional selective neurons.

Neurophysiological studies have revealed that direction selective neurons widely exist in different animal species, including invertebrates and vertebrates, such as fly, beetle, locust, zebrafish, cat, rabbit [29], [46]-[55]. There are many ways to construct a directional selective neural network (DSNN) model[29], [31], [56]. In the recent years, one type of whole-field DSNNs based on asymmetric lateral inhibitory mechanism were modeled [57]-[60], and a large number of experiment results suggest that it is robust in object's motion direction perception.

\section{E. The proposed bio-plausible quantitative model}

In this paper we propose a new quantitative model to perceive rotational motions mainly on the fronto-parallel plane. Based on the asymmetrically inhibited directional selective neuron models, the proposed hierarchical visual neural network has a mechanism of spatiotemporal coherence for rotational motion perception. In this visual neural network, different asymmetric lateral inhibitory mechanisms are used to perceive sixteen motion directions. Similar to the arrangement of directional columns in mammalian's cerebral cortex (see [61]), the sixteen direction selective neurons are arranged in a specific order and forming a cyclic structure to perceive the continuous changes of motion directions. The excitation of each directional selective neuron is delayed with one time step, and then multiplied with the gathered excitation from the neuron and its unilateral counterparts depending on which rotary direction to perceive. Since the gathered excitation is from only one specific side, this rotational motion perception neural network (RMPNN) can respond to either counterclockwise (ccw) or clockwise (cw) rotational motion.

\section{The Rotation PERCEPTION Vision System}

In the study of biological visual system, Morrone et al. provided psychophysical evidence for the existence of neural mechanisms in human vision, and revealed that rotational or radial motions perception includes two stages of visual processing: (i) motion-sensitive neurons in V1 respond best to local translation, and (ii) many neurons in MST have large receptive fields tuned to rotational or radial motion [62]. However, Morrone et al. only mentioned such two phrases from the psychophysics' point of view and did not investigate how neural systems percept rotational motions.

In this study, a RMPNN to perceive rotational motion should be based on their presynaptic and postsynaptic counter parts. The RMPNN includes two types of networks, one is ccwRMPNN, which responds to the 'counter clock wise' (ccw) rotational motion, and the other is cwRMPNN, which responds to the clockwise rotational motion. We chose ccwRMPNN as an example to present its structure and principle in this paper. The schematic illustration of a ccwRMPNN is given in Fig.1 (a).

As shown in the Fig.1(a), the ccwRMPNN consists of two main parts: the first is presynaptic networks which are sixteen whole field DSNNs to perceive translation motion cues, and the second is postsynaptic network which focuses on the perception of rotational motion. Details of the two parts will be given in the following sub-sections.

\section{A. The presynaptic networks for motion direction perception}

The presynaptic networks of ccwRMPNN contains the whole field DSNNs, which are base on the previous works [57]-[60], and they can perceive different visual motion cues on the fronto-parallel plane in the field of view. According to the difference of lateral inhibition regions, the sixteen directional selective neurons in the DSNNs are divided into three types. For all of them, they have the similar structure except different inhibition regions. Therefore we choose three typical direction selective neurons in each type: the left neuron $L$, the upper left directional selective neuron $L U$ and the right region of left directional selective neuron $L_{R}$ as examples to illustrate their information processing mechanism.

The left directional selective neuron ( $L$ neuron) prefers the left direction moving edges on the fronto-parallel plane; its presynaptic neural network is illustrated in the upper part of Fig. 1 (a). As shown in the figure, there are four layers and one neuron in the presynaptic network of $L$ neuron: a $P$ layer, a $E$ / I layer, an $S$ layer and a $L$ neuron. The function of each layer and the directional selective neuron $L$ will be described in detail in the next part.

1) Player

The first layer of the $L$ neuron neural network is the photoreceptor $P$ cells. These cells are arranged in matrix form. In this layer, each photoreceptor cell collects the luminance $L_{f}$ of each pixel in the input image at frame $f$, and calculates

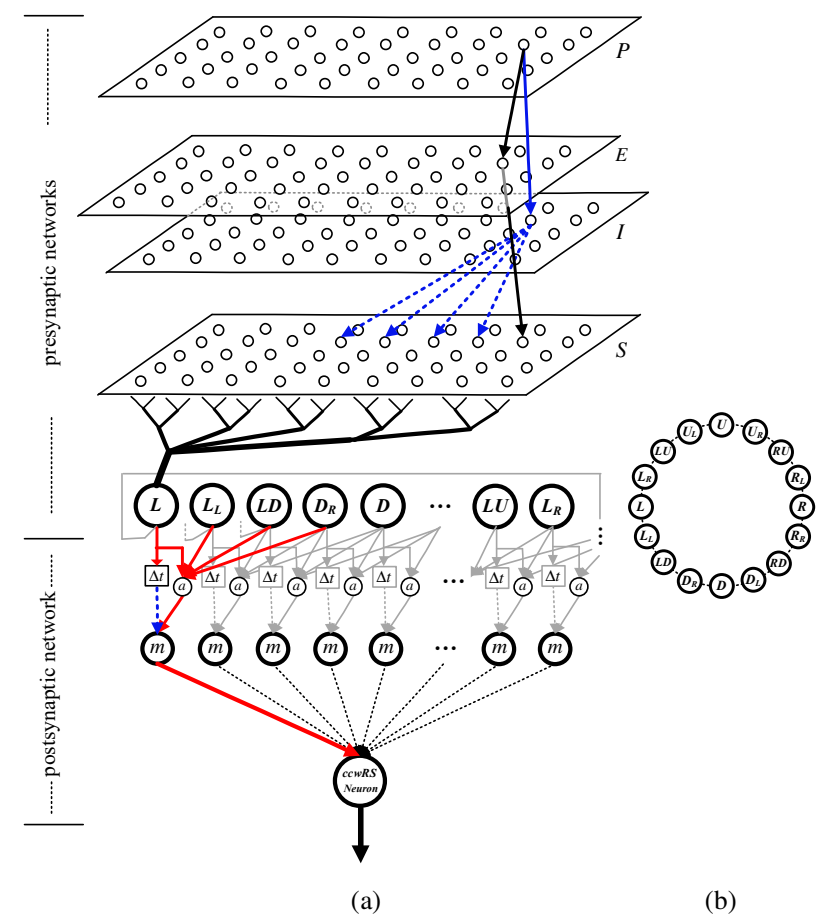

Fig. 1. (a) Schematic illustration of ccwRMPNN. (b) Schematic illustration of the sixteen directional selective neurons' arrangement in ccwRMPNN. 
the change of luminance $P_{f}$ between two sequentially adjacent frames of the video images. Then excitation of these photoreceptor cells as output results of this layer. The output of each cell in this layer is given by

$$
\begin{aligned}
P_{f}(x, y)= & \sum_{i}^{n_{p}} p_{i} P_{f-i}(x, y) \\
& +a b s\left(L_{f}(x, y)-L_{f-1}(x, y)\right)-\xi_{\text {noise }}
\end{aligned}
$$

where, $P_{f}(x, y)$ is the change of the luminance corresponds pixel $(x, y)$ at frame $f ; x$ and $y$ are the pixel coordinates; $p_{i} \in(0,1)$ is the persistence coefficient, it is defined by $p_{i}=\left(1+e^{\mu i}\right)^{-1}, \mu \in(-\infty,+\infty) ; n_{p}$ represents the maximum time steps (or number of image frames) the persistence of the luminance change can last; $L_{f}$ and $L_{f-1}$ are the luminance, subscript $f$ denotes the current frame and $f-1$ denotes the previous frame; $\xi_{\text {noise }}$ is the threshold of signal noise.

\section{2) $I /$ E layer}

The output of the $P$ cells is the input to the next separate two type's cells in the $E$ / I layers respectively. In these two layers, the first type of cells is excitatory cells, through which excitation is passed directly to their retinotopic counterpart in the third layer of the network, the $S$ layer; the second type of cells is inhibition cells, which pass inhibition to their retinotopic counterpart's neighboring cells in the $S$ layer. In both of the $E$ / I layers, cells are arranged in matrix forms.

The excitation and the inhibition from a $P$ cell pass to its counterpart in the $E$ layer and the $I$ layer directly. The input excitation $E(x, y)$ in an $E$ cell and the input inhibition $I(x, y)$ in an $I$ cell have the same value as that in the corresponding $P$ cell.

3) S layer

Cells in the $S$ layer receive excitation and inhibition from the $E$ / I layers, and these cells are also arranged in a matrix form. The output excitation of an $E$ cell is the same as its input excitation, and passes to its counterpart in the $S$ layer directly. However, the output inhibition of an $I$ cell is its input inhibition delay one time step (or image frame), and passes to its retinotopic counterpart's left side neighboring cells (or passes to its counterpart's all neighboring cells except left side, if $L$ cell should prefer leftward movements only) in the $S$ layer up to $n$ cells away. Therefore, the strengths of excitation and gathered inhibition to a cell in $S$ layer are

$$
\begin{aligned}
& E_{f}(x, y)=P_{f}(x, y) \\
& I_{f}^{L}(x, y)=\sum_{i=1}^{n_{\text {inh }}} P_{f-1}(x+i, y) w_{I}(i)
\end{aligned}
$$

where $E_{f}(x, y), I_{f}^{L}(x, y)$ are the excitation and the inhibition to the same cell in the $S$ layer at $(x, y)$ respectively, superscript $L$ denotes the specific directional selective neuron which prefer the left direction motion; $n_{i n h}$ is the inhibition radius, i.e. the maximum number of cells in $I$ layer that spread their inhibitions to the same cell in the $S$ layer at $(x, y)$;
$w_{I}(i) \in[0,5.5]$ is the local inhibition weight which controls the neighboring inhibition strength. Therefore, with an appropriate inhibition radius from the right side with one frame delay, the $S$ cells' excitation caused by the left moving edges can be eliminated or weakened sharply in this layer.

Then, the excitation strength of these cells gathered in a $S$ cell is

$$
S_{f}(x, y)=E_{f}(x, y)-I_{f}^{L}(x, y) W_{I}
$$

where $S_{f}(x, y)$ is the specific cell's excitation in the $S$ layer at $(x, y) ; W_{I}$ is the global inhibition weight which control the overall inhibition strength.

In the $S$ layer, only those cells which excitation exceeds the threshold $T_{r s}$, their excitation will be transmitted to $L$ Neuron. So, if the excitation of a cell is less than the threshold $T_{r s}$, its excitation is set to zero, otherwise remains unchanged.

$$
\tilde{S}_{f}(x, y)= \begin{cases}0, & \text { if } S_{f}(x, y)<T_{r s} \\ S_{f}(x, y), & \text { if } S_{f}(x, y) \geq T_{r s}\end{cases}
$$

4) Left direction selective neuron: L neuron

The input excitation of $L$ neuron is summed by the excitation of all cells in the $S$ layer,

$$
\operatorname{Sum}_{f}^{L}=\sum_{x=1}^{n_{c}} \sum_{y=1}^{n_{r}} \operatorname{abs}\left(\tilde{S}_{f}(x, y)\right)
$$

where, $\operatorname{Sum}_{f}^{L}$ is the summed excitation of the $L$ neuron; $n_{c}$ and $n_{r}$ are the total number of cells in a row and a column in the $S$ layer respectively.

The summed excitation of the $L$ neuron is then processed as

$$
V_{f}^{L}=2 \times\left(1-\left(1+e^{-\frac{\operatorname{Sum}_{f}^{L}}{n_{r c}}}\right)^{-1}\right)
$$

here $n_{r c}$ is the total number of the cells in the $S$ layer. According to (6), $\operatorname{Sum}_{f}^{L}$ is greater than or equal to zero, so $L$ neuron's excitation $V_{f}^{L} \in[0 \sim 1.0]$.

As the spatiotemporal processing mechanism described above, the stimuli generated by the object which moving to left will make $L$ neuron's excitation be the maximum in the sixteen direction selective neurons. Other direction selective neurons, for example the right directional selective neuron $(R)$, the up directional selective neuron $(U)$ and the down directional selective neuron $(D)$, share the same mechanism in forming their direction selective sensitiveness.

5) The Other Types of Direction Selective Neurons

Besides the above $L, R, U$ and $D$ direction selective neurons, two types of direction selective neurons which diagonal visual motion and neighborhood direction visual motion also are used in this paper. There are four neurons to perceive the diagonal visual motions: $L U$ (left-up), $L D$ (left-down), $R U$ (right-up), $R D$ (right-down), and eight neurons to perceive the neighborhood direction visual motions: $L_{L}$ (left side of $L$ ), $L_{R}$ (right side of $\left.L\right), D_{L}$ (left side of $D$ ), $D_{R}$ (right side of $\left.D\right), R_{L}$ (left side of $R$ ), $R_{R}$ (right side of $R$ ), $U_{L}$ (left side of $U$ ), $U_{R}$ (right side of $U$ ). 

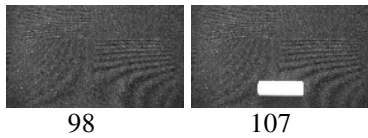

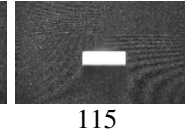

(a)

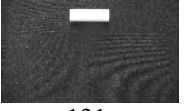

131

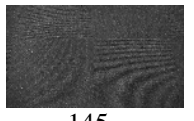

145

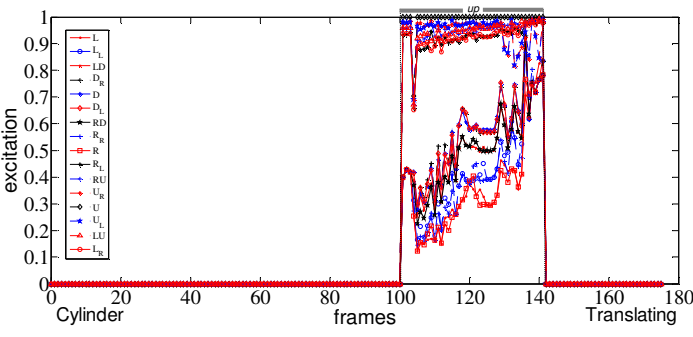

(b)

Fig. 2. An example of direction selective neurons process video sequences with an up moving white cylinder on a carpeted office floor. (a) Sample images of the video sequence; the frame number is indicated under each image. (b) Excitation of the sixteen direction selective neurons.

We take $L U$ neuron and $L_{L}$ neuron as examples to describe the two types' direction perception principle. The differences between $L U, L_{L}$ and $L$ are the regions of inhibition direction in $S$ layer. For $L U$ neuron, the inhibition from an $I$ cell passes to its retinotopic counterpart's 'left-up' side neighboring cells in the $S$ layer up to $n$ cells away with one image frame delay. The gathered strength of inhibition to a cell in this $S$ layer is

$$
I_{f}^{L U}(x, y)=\sum_{j=1, i=j}^{n_{i n h}} P_{f-1}(x+i, y+j) w_{I}(i, j)
$$

Similarly, for $L_{L}$ neuron, the gathered strength of inhibition to a cell in the $S$ layer is

$$
I_{f}^{L_{L}}(x, y)=\sum_{i=2, j=1, i>j}^{n_{\text {inh }}} P_{f-1}(x+i, y-j) w_{I}(i, j)
$$

For other diagonal and neighborhood direction selective neurons, the inhibition gathered by a cell in $S$ layer can be illustrated in the similar way.

6) The arrangement of the sixteen direction selective neurons

In the cerebral cortex of mammalian, neurons with a similar axis of motion preference exist in the form of directional columns, which may represent directions continuously from 0 to 360 degree [61]. Similarly, the sixteen direction selective neurons in RMPNN's presynaptic network are arranged in this specific order and forming a cyclic structure to perceive different motion direction (as indicated in Fig. 1 (b)). Consequently, at any given time, the whole presynaptic networks are able to perceive the changing motion directions of an object, and pass these motion cues to their postsynaptic network for further processing.

To demonstrate the functionality of these presynaptic networks in detecting motion direction, one video sequence showing an up moving white cylinder on a carpeted office floor was processed by the sixteen DSNNs. As shown in Fig.2, the responded excitation from the directional selective neuron $U$, and other neurons close to $U$, are significantly greater than that from other direction selective neurons. This experiment reassured that the visual motion cues can be extracted by these DSNNs for further processing.

\section{B. The postsynaptic network for rotational motion perception}

The schematic illustration of ccwRMPNN's postsynaptic networks is shown in the lower part of Fig. 1 (a). In RMPNN's postsynaptic network, the excitation of each directional selective neuron is delayed with one time step and then multiplied with the gathered excitation from the neuron and its unilateral counterparts depending on which rotary direction to perceive (e.g., right side for ccwRMPNN, and left side for cwRMPNN). The postsynaptic networks consist of two layers and one specific rotational motion selective neuron: an excitation gathering operation layer, an excitation multiplication operation layer and a counterclockwise rotation selective (ccwRS) neuron.

Expression $\Gamma_{f}$ is the sixteen direction selective neurons' excitations vector in ccwRMPNN's presynaptic networks.

$$
\Gamma_{f}=\left(V_{f}^{L} V_{f}^{L_{L}} V_{f}^{L D} V_{f}^{D_{R}} V_{f}^{D} \ldots V_{f}^{L_{R}}\right)^{T}
$$

where subscript $f$ denotes time step (or video frame number); and $V_{f}^{L}, V_{f}^{L_{L}}, V_{f}^{L D}, V_{f}^{D_{R}}, V_{f}^{D}, \ldots V_{f}^{L_{R}}$ are the excitations of the sixteen direction selective neurons.

Excitation of each directional selective neuron which spread into the postsynaptic network is determined by a spiking mechanism. When the excitation of a directional selective neuron $V_{f}^{i}$ exceeds threshold $T_{e}$, one internal spike occurs inside this neuron,

$$
S_{f}^{\text {Transpike }}(i)=\left\{\begin{array}{cc}
1, & \text { if } V_{f}^{i}>T_{e} \text { and } V_{f}^{i}>0 \\
0, & \text { otherwise }
\end{array}\right.
$$

If there are $m_{s p}$ spikes in $m_{t s}$ time steps $\left(m_{s p} \leq m_{t s}\right)$ inside the same directional selective neuron $q\left(q \in\left\{L, \ldots, L_{R}\right\}\right)$, a non-rotation is perceived. Simultaneously, only excitation which exceeds or equal to threshold $T_{e}$, their excitations will be transmitted to their retinotopic counterpart in the postsynaptic network.

$$
\begin{gathered}
\tilde{V}_{f}(i)=\left\{\begin{array}{l}
0, \quad \text { if } V_{f}(i)<T_{e} \text { or } \sum_{j=0}^{m_{t s}-1} S_{f-j}^{\text {Transpikes }}(q) \geq m_{s p} \\
V, \quad \text { if } V_{f}(i) \geq T_{e} \text { and } \sum_{j=0}^{m_{t s}-1} S_{f-j}^{\text {Transpikes }}(q)<m_{s p}
\end{array}\right. \\
V_{f}(i) \in\left\{V_{f}^{L}, V_{f}^{L_{L}}, V_{f}^{L D}, V_{f}^{D_{R}}, V_{f}^{D}, \ldots, V_{f}^{L_{R}}\right\}
\end{gathered}
$$

In this formula, the threshold $T_{e}$ is defined by the following

$$
T_{e}=\underset{i=1, \ldots, n}{\operatorname{MAX}} \underset{2 n d}{ }\left\{V_{f}(i)\right\}
$$

where $\operatorname{MAX}_{i=1}\left\{V_{f}(i)\right\}$ represents the sub-maximum in the 
array $V_{f}(i)$.

Therefore, the output excitations vector of the sixteen direction selective neurons spread into the postsynaptic network is

$$
\tilde{\Gamma}_{f}=\left(\begin{array}{lllllll}
\tilde{V}_{f}^{L} & \tilde{V}_{f}^{L_{L}} & \tilde{V}_{f}^{L D} & \tilde{V}_{f}^{D_{R}} & \tilde{V}_{f}^{D} \ldots \tilde{V}_{f}^{L_{R}}
\end{array}\right)^{T}
$$

For a stationary object, all elements in the vector $\tilde{\Gamma}_{f}$ are zero. When the object is in motion, however, a few elements in $\tilde{\Gamma}_{f}$ could be greater than zero. The function of each layer and the ccwRS neuron will be described below in detail.

1) Excitations Gathering Operation Layer

The first layer of the postsynaptic network is Excitation Gathering Operation layer, and it consists of sixteen identical cells $a$. Each $a$ cell receives excitations from its ccw side neighboring direction selective neurons in the upper layer up to $m_{\text {neighbor }}$ neurons away. Therefore, the strengths of excitation to an $a$ cell in this layer are

$$
A_{f}(i)=\underset{j=0, . ., m_{\text {neighbor }}}{\operatorname{MAX}}\left\{\widetilde{\Gamma}_{f}(i+j)\right\}
$$

where $A_{f}(i)$ is the gathered excitation of an $a$ cell, $i$ denotes the index of an $a$ cell, and subscript $f$ denotes the current frame; $m_{\text {neighbor }}$ is the maximum number of directional selective neuron in the upper layer that spread excitation to an $a$ cell in this layer; $\widetilde{\Gamma}_{f}(i+j)$ is the $(i+j)$ th index direction selective neuron's excitation in the upper layer. From the above, $A_{f}(i)$ is equal to either zero or the gathered excitations from the (i) th to the $\left(i+m_{\text {neighbor }}\right)$ th direction selective neurons in the current frame.

\section{2) Excitations Multiplication Operation Layer}

The postsynaptic network's second layer is Excitations Multiplication Operation layer; it is composed of sixteen identical multiplication operator cells $m$. These $m$ cells receive the excitation from its retinotopic counterpart in the sixteen direction selective neurons with one frame delay, and then multiply this excitation with the other excitation transmitted by its retinotopic counterpart in the excitation gathering operation layer immediately. Therefore, the gathered excitations to a $m$ cell in this layer is

$$
M_{f}(i)=A_{f}(i) \times V_{f-1}^{\sim}(i)
$$

where $M_{f}(i)$ is the multiplication result of a $m$ cell's excitation, $i$ denotes the index of the sixteen $m$ cells, subscript $f$ denotes the current frame and $f-1$ denotes the previous frame. From the above, $M_{f}(i)$ is great than or equal to zero.

\section{3) ccwRS Neuron}

Finally, sixteen $m$ cells' excitations are converged to ccwRS neuron. The strength of the converged excitations to the ccwRS neuron is

$$
\kappa_{f}=\underset{i=1, \ldots, n}{\operatorname{MAX}}\left\{M_{f}(i)\right\}
$$

where $\kappa_{f}$ is the input excitation of ccwRS neuron, subscript $f$ denotes the current frame; $n$ is the number of the

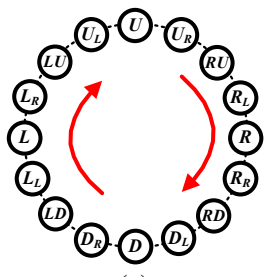

(a)

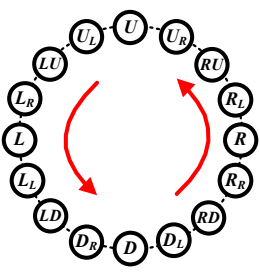

(b)
Fig. 3. The only difference between cwRMPNN and ccwRMPNN is the opposite excitation transmission direction sequence of sixteen directional selective neurons. (a) cwRMPNN. (b) ccwRMPNN.

TABLE I

PARAMETERS OF RMPNN

\begin{tabular}{cccc}
\hline \hline Name & Value & Name & Value \\
\hline$p_{i}$ & 0 & $\sigma$ & 0.5 \\
$u$ & 0 & $n_{\text {inh }}$ & $8 \sim 30$ \\
$w_{I}$ & 5.5 & $m_{\text {neighbor }}$ & 3 \\
$W_{I}$ & 1.7 & $m_{s p}$ & 6 \\
$T_{r s}$ & 12 & $m_{t s}$ & 6 \\
$n_{r c}$ & 11200 & $n_{t s}$ & 8 \\
$n_{c}$ & 140 & $T_{s}$ & 0 \\
$n_{r}$ & 80 & & \\
\hline \hline
\end{tabular}

$m$ cells.

The output excitation of the ccwRS neuron is regulated by a spiking mechanism, i.e., when the input excitation $\kappa_{f}$ exceeds a threshold $T_{s}$, an internal spike inside the ccwRS neuron is produced otherwise remain silent,

$$
S_{f}^{R S N \text { spike }}= \begin{cases}1, & \text { if } \kappa_{f}>T_{s} \\ 0, & \text { otherwise }\end{cases}
$$

If there are $n_{s p}$ successive spikes occurring inside the ccwRS neuron, a ccw rotation motion is perceived, and the ccwRS neuron will produce its output excitation immediately. If $\kappa_{f} \geq \gamma_{a m}\left(\gamma_{a m}=0.9\right)$, the output excitation $\widetilde{\kappa}_{f}=\kappa_{f}$, otherwise $\widetilde{\kappa}_{f}$ is encouraged iteratively by the following equation till it is greater than or equal to $\gamma_{a m}$,

$$
\tilde{\kappa}_{f}=\kappa_{f} \times \sigma^{\kappa_{f}-1}
$$

here $\sigma$ is the excitation amplification factor. After being encouraged, the $\widetilde{\kappa}_{f} \in[0.9 \sim 1]$. Finally, the output excitation of the ccwRS neuron $F_{c c w R S}$ is

$$
F_{c c w R S}=\left\{\begin{aligned}
\tilde{\kappa}_{f}, & \text { if } \sum_{f_{s}}^{f} s_{f}^{R S N \text { spike }} \geq n_{s p} \\
0, & \text { otherwise }
\end{aligned}\right.
$$

The threshold $n_{s p}$ is

$$
n_{s p}=n_{t s}+\sum_{f^{1}=f_{s}}^{f}\left(m_{f^{1}}^{s}(k)-\frac{m_{\left(f^{1}-1\right)}^{s}(k) !}{m_{\left(f^{1}-2\right)}^{s}(k) !}\right)
$$




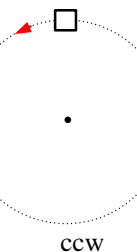

(a)

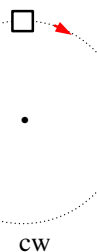

(b)

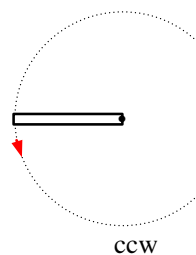

(c)

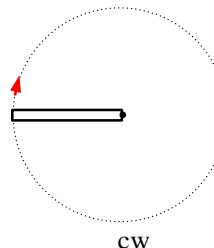

(d)

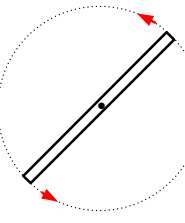

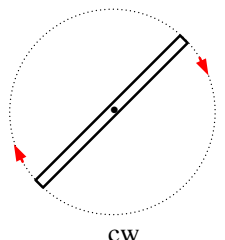

(f)

Fig. 4. Schematic illustrations of three types of objects' rotational motion patterns. (a) A ccw rotating block. (b) A cw rotating block. (c) A ccw rotating half-bar. (d) A cw rotating half-bar. (e) A ccw rotating bar. (f) A cw rotating bar. All video sequences are accessible at http://www.ciluk.org/temp/05_TestVideos.zip.
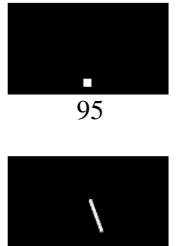

137

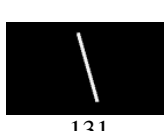

131
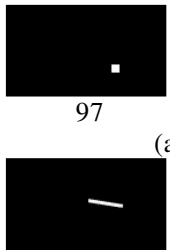

139

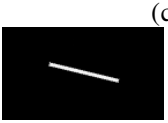

133

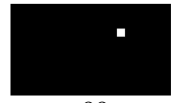

99

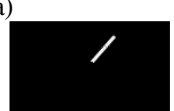

141

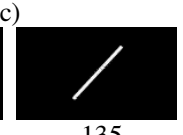

135

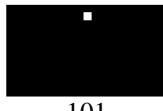

101

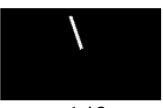

143

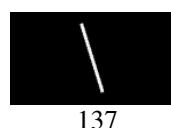

137

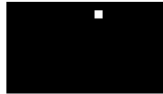

120

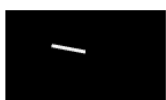

199

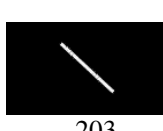

203

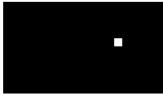

122

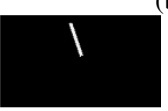

201

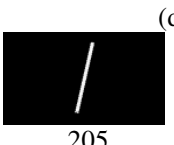

205

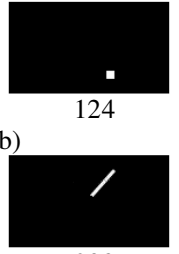

203

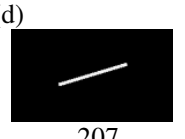

(f)
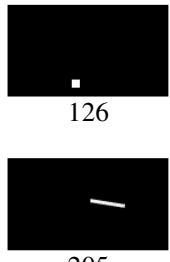

205

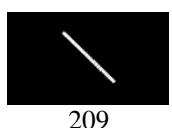

209

Fig. 5. Example frames from the simulated visual stimuli tests. Each video sequence is represented with four frames; the frame number is indicated under each image. (a) A ccw rotating $7 \times 7$ pixels white block. (b) A cw rotating $7 \times 7$ pixels white block. (c) A ccw rotating $3 \times 30$ pixels white half-bar. (d) A cw rotating $3 \times 30$ pixels white half-bar. (e) A ccw rotating $3 \times 60$ pixels white bar. (f) A cw rotating $3 \times 60$ pixels white bar. (Detailed experiment settings and results see Table II.)

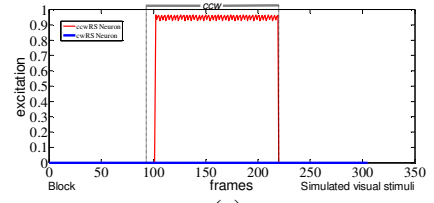

(a)

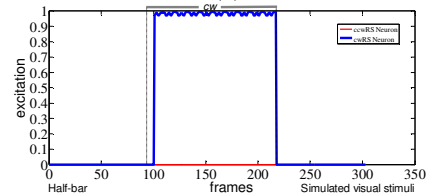

(d)

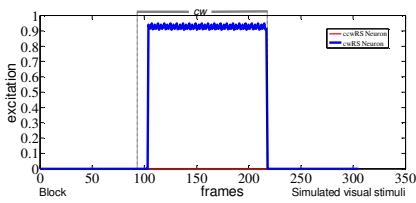

(b)

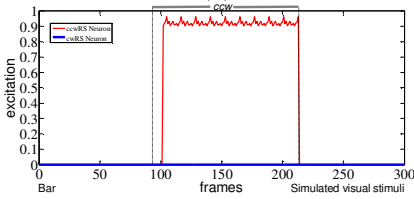

(e)

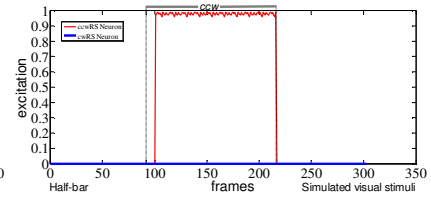

(c)

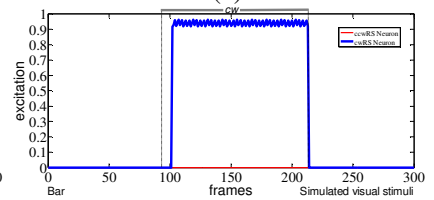

(f)

Fig. 6. Output curves of ccwRS neuron and cwRS neuron's excitation. Each sub-graph uniquely corresponds to the experimental results of the same identifier video sequence in Fig. 5.

where $k\left(k \in\left\{L, \ldots, L_{R}\right\}\right)$ denotes the index of the directional selective neuron which produces a spike at frame $f^{1}$, $m_{f^{1}}^{s}(k)\left(0 \leq m_{f^{1}}^{s}(k) \leq m_{s p-1}\right)$ is the total number of continuous spikes occurring inside the directional selective neuron $k$ up to frame $f^{1}$. The $m_{f^{1}}^{s}(k)$ can be summarized as

$$
m_{f}^{s}(k)=\left\{\begin{array}{lr}
\sum_{i=0}^{m_{t s}-2} S_{f-1}^{\text {Transpike }}(k), & \text { if } f \geq f_{s} \\
0, & \text { s.t. } f-i \geq f_{s} \\
& \text { otherwise }
\end{array}\right.
$$

here $f_{s}$ denotes the first time step (or image frame) of the current time period when continuous spikes are occurring inside the ccwRS neuron.

\section{The $\mathrm{cw}$ rotational motion perception neural network}

The structure of a cwRMPNN is the same as the above described structure of a ccwRMPNN, however the excitation gathering direction from these direction selective neurons is completely the opposite, as shown in Fig.3. In ccwRMPNN, when a ccw rotational motion occurs, the excitations of direction selective neurons are successively transmitted in accordance with the $\mathrm{ccw}$ direction, and the ccw rotational motion is perceived by ccwRMPNN. However, a ccwRMPNN will not respond to a cw rotational motion, unless its internal structure in gathering excitations are changed to an opposite way - i.e., to become a cwRMPNN (see Fig. 3).

\section{Parameters of the System}

In this study, all experiments are executed on a Microsoft Windows Server 2008 with CPU/2.66G and RAM/4G, source 
TABLE II

Rotational Motion Region Perceptions in SimUlated Visual Stimuli Tests (EXPERIMENTS SEe Fig.5 AND 6)

\begin{tabular}{|c|c|c|c|c|c|c|c|c|c|c|c|}
\hline Video & $\begin{array}{l}\text { The } \\
\text { total of } \\
\text { frames }\end{array}$ & $\begin{array}{l}\text { Object } \\
\text { type }\end{array}$ & $\begin{array}{c}\text { Practical } \\
\text { ccw } \\
\text { rotational } \\
\text { motion } \\
\text { region } \\
\text { (frames) } \\
\end{array}$ & $\begin{array}{c}\text { Practical } \\
\text { cw } \\
\text { rotational } \\
\text { motion } \\
\text { region } \\
\text { (frames) } \\
\end{array}$ & $\begin{array}{l}\text { Angular } \\
\text { velocity }\end{array}$ & $\begin{array}{c}\text { RMPNN's } \\
\text { ccw } \\
\text { rotational } \\
\text { motion } \\
\text { region } \\
\text { (frames) } \\
\end{array}$ & $\begin{array}{c}\text { RMPNN's } \\
\text { cw } \\
\text { rotational } \\
\text { motion } \\
\text { region } \\
\text { (frames) } \\
\end{array}$ & $\begin{array}{c}\text { ccwRS } \\
\text { neuron's } \\
\text { success rate } \\
(\%)\end{array}$ & $\begin{array}{c}\text { ccwRS } \\
\text { neuron's false } \\
\text { alarm rate } \\
(\%)\end{array}$ & $\begin{array}{c}\text { cwRS } \\
\text { neuron's } \\
\text { success rate } \\
(\%)\end{array}$ & $\begin{array}{c}\text { cwRS } \\
\text { neuron's false } \\
\text { alarm rate } \\
(\%)\end{array}$ \\
\hline $\mathrm{a}$ & 306 & block & $93-219$ & N/A & $15.7 \mathrm{rad} / \mathrm{s}$ & $102-219$ & N/A & 100 & N/A & N/A & 0 \\
\hline $\mathrm{b}$ & 306 & block & N/A & $93-217$ & $15.7 \mathrm{rad} / \mathrm{s}$ & N/A & $104-217$ & N/A & 0 & 100 & N/A \\
\hline $\mathrm{c}$ & 304 & half-bar & $92-216$ & N/A & $15.7 \mathrm{rad} / \mathrm{s}$ & $101-216$ & N/A & 100 & N/A & N/A & 0 \\
\hline d & 304 & half-bar & N/A & $93-217$ & $15.7 \mathrm{rad} / \mathrm{s}$ & N/A & $101-217$ & N/A & 0 & 100 & N/A \\
\hline $\mathrm{e}$ & 301 & bar & $93-213$ & N/A & $15.7 \mathrm{rad} / \mathrm{s}$ & $102-213$ & N/A & 100 & N/A & N/A & 0 \\
\hline f & 301 & bar & N/A & $93-213$ & $15.7 \mathrm{rad} / \mathrm{s}$ & N/A & $102-213$ & N/A & 0 & 100 & N/A \\
\hline
\end{tabular}

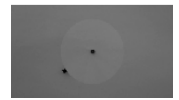

166

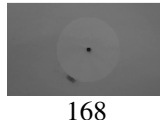

168

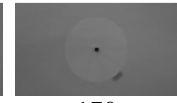

170

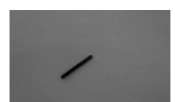

165

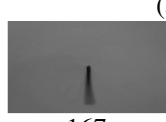

167

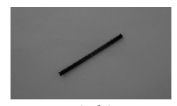

161

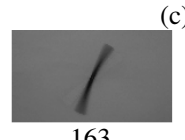

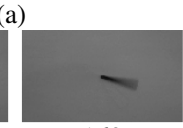

169

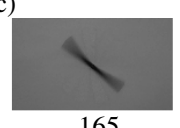

165

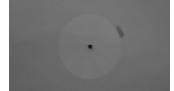

172

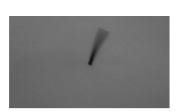

171

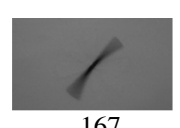

167

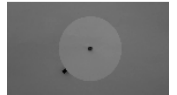

169

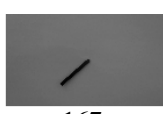

167

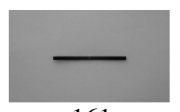

161

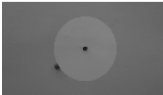

171

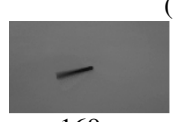

169

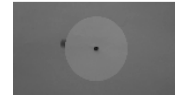

(b)
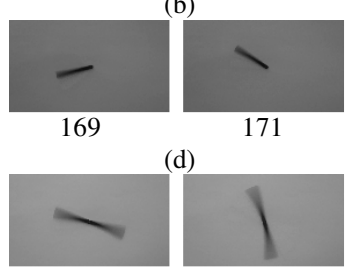

163

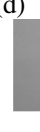

(f)
173

171

165
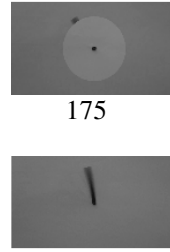

173

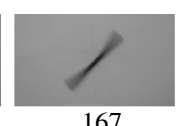

167

Fig. 7. Example frames from the real scene video tests. Each video sequence is represented with four frames; the frame number is indicated under each image. (a) A ccw rotating black block. (b) A cw rotating black block. (c) A ccw rotating black half-bar. (d) A cw rotating black half-bar. (e) A ccw rotating black bar. (f) A ccw rotating black bar. (Detailed experiment settings and results see Table III.)

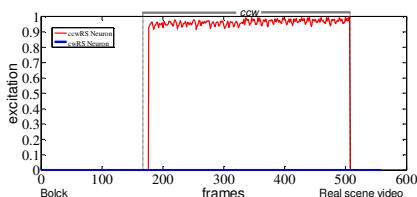

(a)

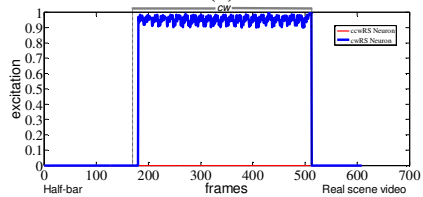

(d)

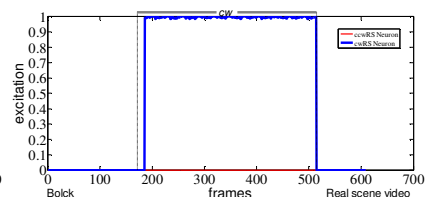

(b)

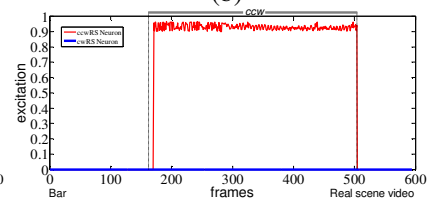

(e)

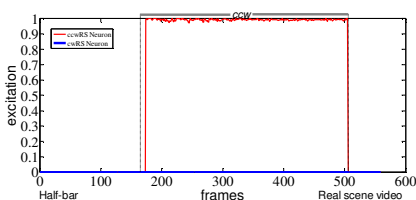

(c)

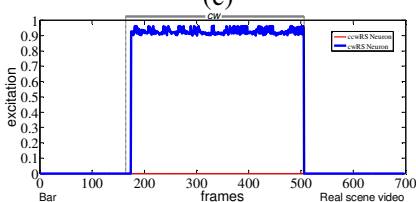

(f)

Fig. 8. Output curves of ccwRS neuron and cwRS neuron's excitation. Each sub-graph uniquely corresponds to the experimental results of the same identifier video sequence in Fig. 7.

codes are written in $\mathrm{C}++$. In the simulation, each real scene video sequences of rotational motion situation taken at 30 frames per second, and the input frames to RMPNN are $140 \times 80$ resolution grayscale images with 8 -bit precision.

Based on the previous experimental studies [63], [57]-[60] and the current trials, the parameters of RMPNN are given in Table I. In these experiments, the direction selective neurons be used are: left selective neuron $L$, right selective neuron $R$, up selective neuron $U$, down selective neuron $D$, left-up selective neuron $L U$, left-down selective neuron $L D$, right-up selective neuron $R U$, right-down selective neuron $R D$, left side of $L$ selective neuron $L_{L}$, right side of $L$ selective neuron
$L_{R}$, left side of $D$ selective neuron $D_{L}$, right side of $D$ selective neuron $D_{R}$, left side of $R$ selective neuron $R_{L}$, right side of $R$ selective neuron $R_{R}$, left side of $U$ selective neuron $U_{L}$, right side of $U$ selective neuron $U_{R}$. All the direction selective neurons used in each experiment are set in the same way except the inhibited directions.

In a RMPNN, each pixel in an input frame image has one corresponding cell in the $P$ layer. Each input frame image is $140 \times 80$ pixels; hence there are 11200 cells in the $P$ layer. It follows from this that there are $11200 E$ cells and 11200 $I$ cells all shared by its sixteen direction selective neurons; it 
TABLE III

Rotational Motion Region PeRceptions In REAL ScEne Video TeSts (EXPERIMENTS SEe FIG.7 AND 8)

\begin{tabular}{|c|c|c|c|c|c|c|c|c|c|c|c|}
\hline Video & $\begin{array}{l}\text { The } \\
\text { total of } \\
\text { frames }\end{array}$ & $\begin{array}{l}\text { Object } \\
\text { type }\end{array}$ & $\begin{array}{c}\text { Practical } \\
\text { ccw } \\
\text { rotational } \\
\text { motion } \\
\text { region } \\
\text { (frames) } \\
\end{array}$ & $\begin{array}{c}\text { Practical } \\
\text { cw } \\
\text { rotational } \\
\text { motion } \\
\text { region } \\
\text { (frames) } \\
\end{array}$ & $\begin{array}{l}\text { Angular } \\
\text { velocity }\end{array}$ & $\begin{array}{c}\text { RMPNN's } \\
\text { ccw } \\
\text { rotational } \\
\text { motion } \\
\text { region } \\
\text { (frames) } \\
\end{array}$ & $\begin{array}{c}\text { RMPNN's } \\
\text { cw } \\
\text { rotational } \\
\text { motion } \\
\text { region } \\
\text { (frames) } \\
\end{array}$ & $\begin{array}{c}\text { ccwRS } \\
\text { neuron's } \\
\text { success rate } \\
(\%)\end{array}$ & $\begin{array}{l}\text { ccwRS } \\
\text { neuron's false } \\
\text { alarm rate } \\
(\%)\end{array}$ & $\begin{array}{c}\text { cwRS } \\
\text { neuron's } \\
\text { success rate } \\
(\%)\end{array}$ & $\begin{array}{c}\text { cwRS } \\
\text { neuron's false } \\
\text { alarm rate } \\
(\%)\end{array}$ \\
\hline $\mathrm{a}$ & 561 & block & $167-507$ & N/A & $\begin{array}{c}23.55 \sim 17.13 \\
\mathrm{rad} / \mathrm{s}\end{array}$ & $177-507$ & N/A & 100 & N/A & N/A & 0 \\
\hline $\mathrm{b}$ & 610 & block & N/A & $171-514$ & $12.99 \mathrm{rad} / \mathrm{s}$ & N/A & $186-514$ & N/A & 0 & 100 & N/A \\
\hline $\mathrm{c}$ & 561 & half-bar & $165-505$ & N/A & $\begin{array}{c}23.55 \sim 17.13 \\
\mathrm{rad} / \mathrm{s}\end{array}$ & $174-505$ & N/A & 100 & N/A & N/A & 0 \\
\hline d & 610 & half-bar & N/A & $169-512$ & $12.99 \mathrm{rad} / \mathrm{s}$ & N/A & $181-512$ & N/A & 0 & 100 & N/A \\
\hline $\mathrm{e}$ & 596 & bar & $162-504$ & N/A & $\begin{array}{c}23.55 \sim 18.84 \\
\mathrm{rad} / \mathrm{s}\end{array}$ & $171-504$ & N/A & 100 & N/A & N/A & 0 \\
\hline $\mathrm{f}$ & 699 & bar & N/A & $164-505$ & $\begin{array}{c}16.38 \sim 15.7 \\
\mathrm{rad} / \mathrm{s}\end{array}$ & N/A & $175-505$ & N/A & 0 & 100 & N/A \\
\hline
\end{tabular}

Fig. 9. Example frames from the varied inhibition radius tests. Each video sequence is represented with four frames; the frame number is indicated under each image. (a) A ccw rotating black half-bar. (b) A cw rotating black bar.

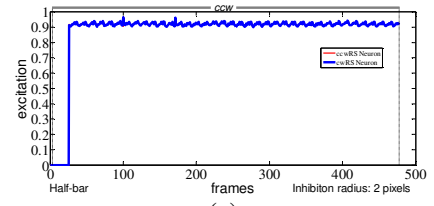

(a)

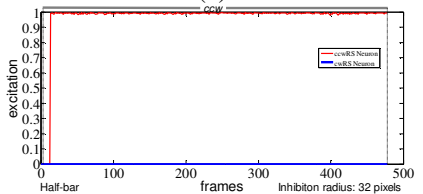

(e)

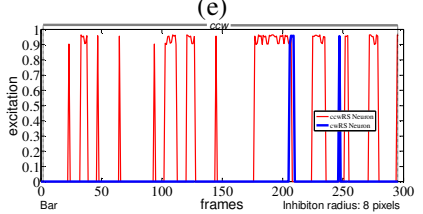

(i)

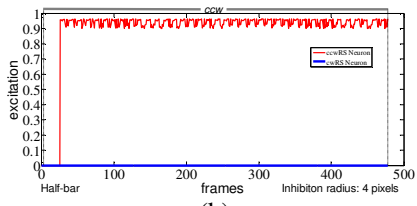

(b)

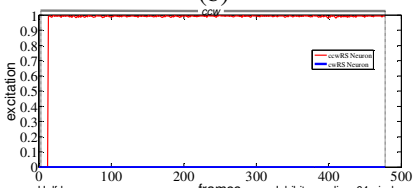

(f)

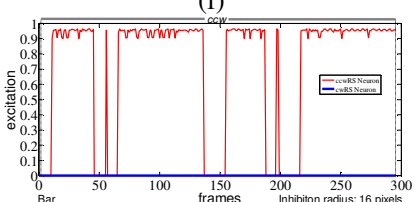

(j)

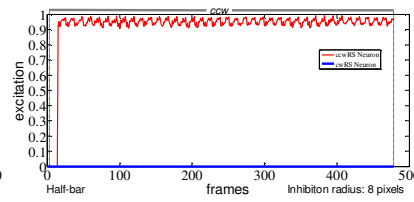

(c)

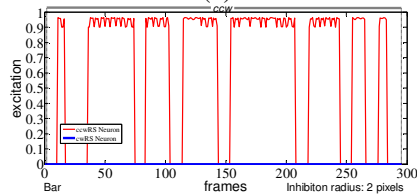

(g)

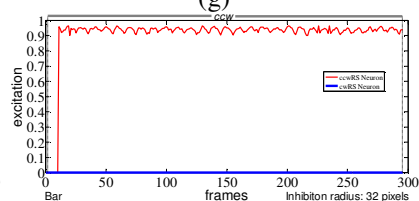

(k)

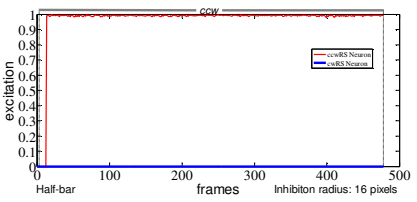

(d)

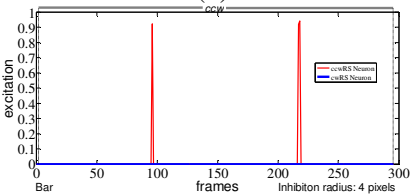

(h)

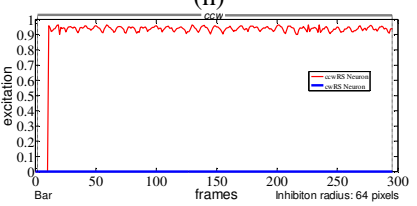

(1)

Fig. 10. Output curves of ccwRS neuron and cwRS neuron's excitation. Each sub-graph uniquely corresponded to a specific inhibition radius value test results of a video sequence in Fig. 9 (a) or (b). (a) Half-bar: $n_{i n h}=2$ pixels. (b) Half-bar: $n_{i n h}=4$ pixels. (c) Half-bar: $n_{i n h}=8$ pixels. (d) Half-bar: $n_{i n h}=16$ pixels. (e) Half-bar: $n_{i n h}=32$ pixels. (f) Half-bar: $n_{i n h}=64$ pixels. (g) Bar: $n_{i n h}=2$ pixels. (h) Bar: $n_{i n h}=4$ pixels. (i) Bar: $n_{i n h}=8$ pixels. (j) Bar: $n_{i n h}=16$ pixels. (k) Bar: $n_{i n h}=32$ pixels. (l) Bar: $n_{i n h}=64$ pixels. Here $n_{i n h}$ is the inhibition radius.

has $179,200 S$ cells and 49 functional cells. So the total number of cells involved in RMPNN is 212,849 .

\section{EXPERIMENTAL STUDY}

In the study of the rotational motion selective neurons of primates' cerebral cortex, it was found these neurons respond well to the rotational motion of a half-bar, or a bar, or a spot [5], [6], [8], [10], [11], [21], [43]. Hence we use several sets of video sequences, which are similar to those rotary objects in the above neurophysiological experiments, to test the performance of RMPNN. The schematics of different rotary objects are

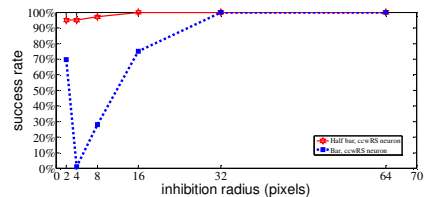

(a)

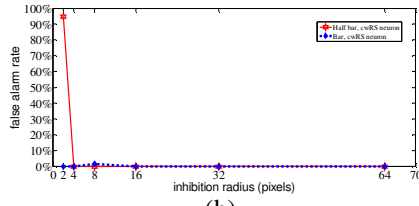

(b)
Fig. 11. CcwRS neuron's perception success rates and cwRS neuron's false alarm rates' curves in the varied inhibition radius tests. (a) The perception success rates of the ccwRS neuron. (b) The perception false alarm rates of the cwRS neuron.

shown in Fig. 4. 


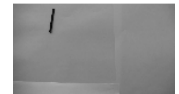

152

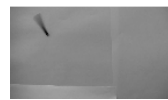

154

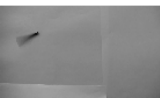

156

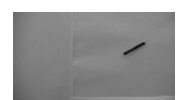

160

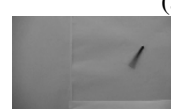

162

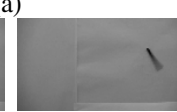

164

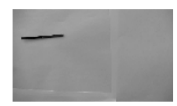

158

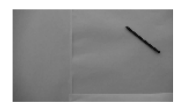

163

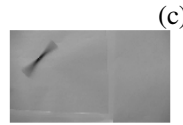

160

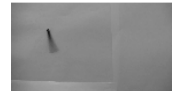

158

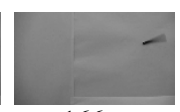

166

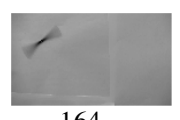

164

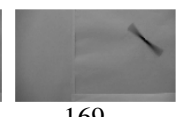

169

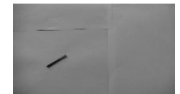

150

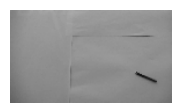

169

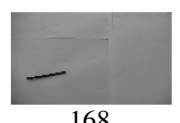

168

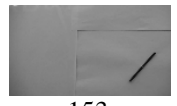

153

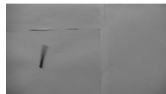

152

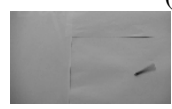

171

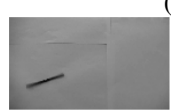

170

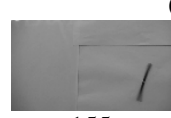

155

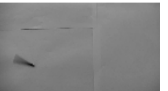

154 (b)

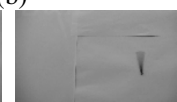

173

(d)

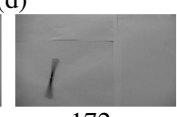

172

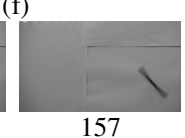

(h)

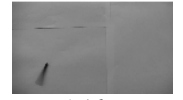

156

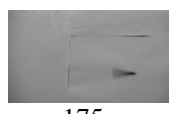

175

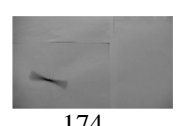

174

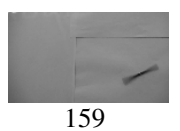

Fig. 12. Example frames from the position invariance and varied receptive field tests. Each video sequence is represented with four frames; the frame number is indicated under each image. (a) Half-bar: top-left area. (b) Half-bar: bottom-left area. (c) Half-bar: top-right area. (d) Half-bar: bottom-right area. (e) Bar: top-left area. (f) Bar: bottom-left area. (g) Bar: top-right area. (h) Bar: bottom-right area.

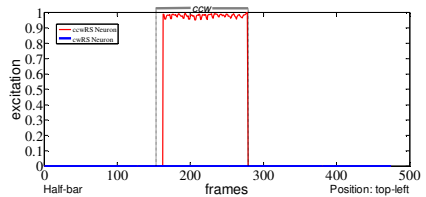

(a)

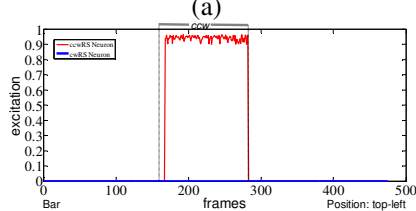

(e)

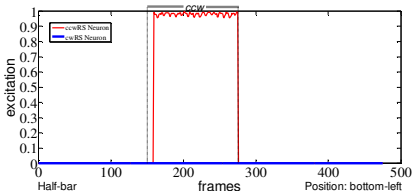

(b)

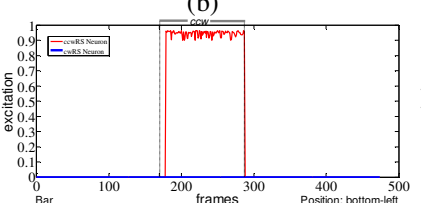

(f)

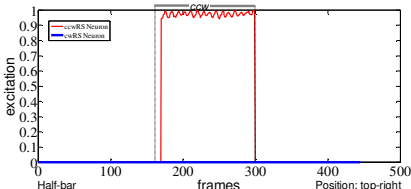

(c)

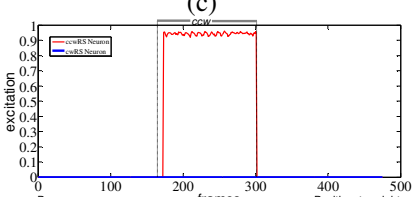

(g)

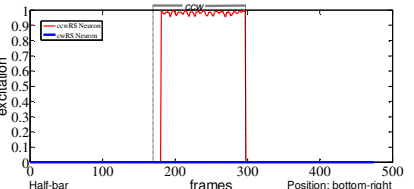

(d)

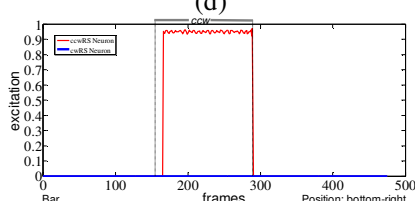

(h)

Fig. 13. Output curves of ccwRS neuron and cwRS neuron's excitation. Each sub-graph uniquely corresponds to the experimental results of the same identifier video sequence in Fig. 12.

In order to test the effectiveness and the robustness of RMPNN, we will use three sets of video sequences in our

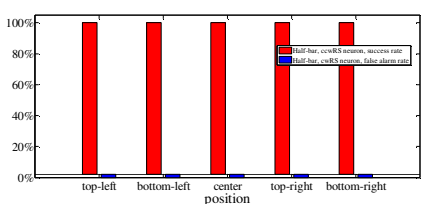

(a)

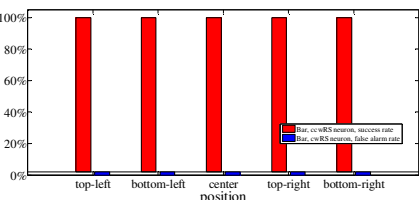

(b)
Fig. 14. CcwRS neuron's perception success rates and cwRS neuron's false alarm rates' histograms in the position invariance and varied receptive field tests. (a) The perception success rates of the ccwRS neuron. (b) The perception false alarm rates of the cwRS neuron.

experiments. The first set of video sequences are computer generated visual stimuli, which simulate the rotational motions of a block, a half-bar and a bar respectively (see Fig. 5). The second set of video sequences are recorded real scene video sequences about the rotational motions of a block, a half-bar and a bar on the fronto-parallel plane (see Fig. 7). The third set of video sequences are the boundary condition tests of the real objects' rotational motion scenes, each of them represents a special real scene (see Fig. 9, 12, 16, 19, 21 and 29). Two indicators were used as the main bases for evaluation in these tests to characterize the performance of RMPNN: the perception success rate of RS neuron and the false alarm rate of RS neuron.

\section{A. Simulated Visual Stimuli Tests}

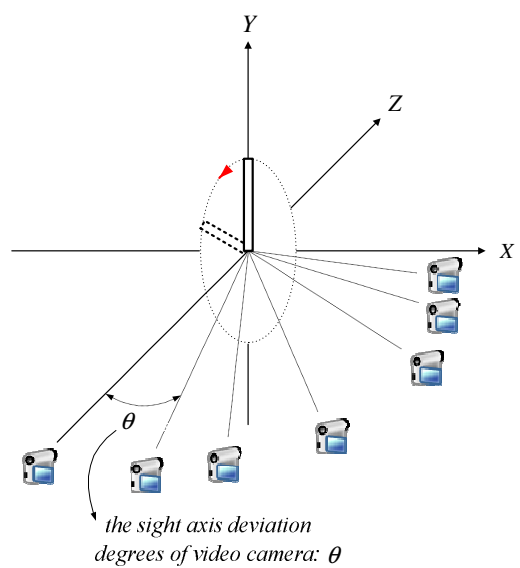

Fig. 15. Schematic illustration of the sight axis deviation tests

In simulated visual stimuli tests, we use six groups of computer generated video sequences - each represents the simulated rotation motion of one of the three objects (e.g. Fig 5, a block, a half-bar, and a bar). The rotational objects are represented with $7 \times 7$ pixels white block (306 frames), $3 \times 30$ pixels white half-bar (304 frames) and $3 \times 60$ pixels white bar (301 frames) conducting $\mathrm{ccw}$ or $\mathrm{cw}$ rotation against black background $(140 \times 80$ pixels $)$ respectively. In all these 


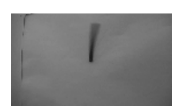

177

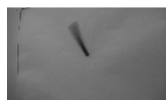

178

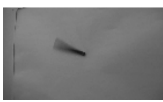

179

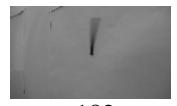

182

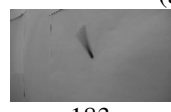

183

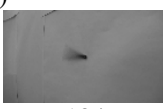

184

(c)

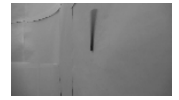

150

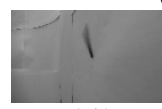

151

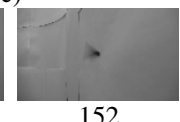

152

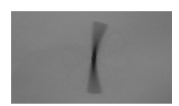

154

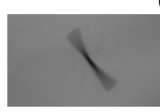

155

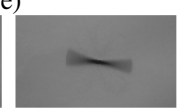

156

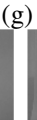

161

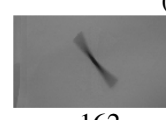

162

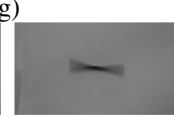

163

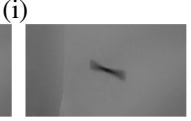

161

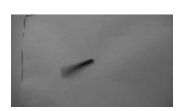

180

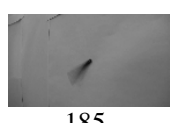

185

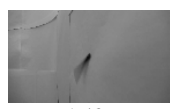

153

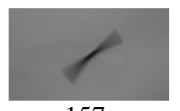

157

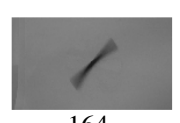

164

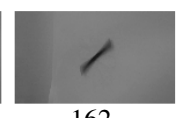

162

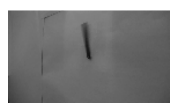

144

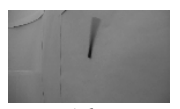

167

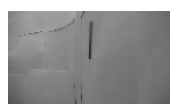

186

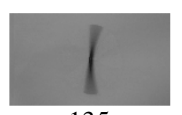

135

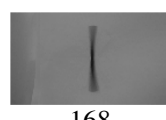

168

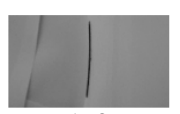

178

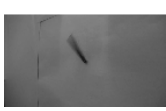

145
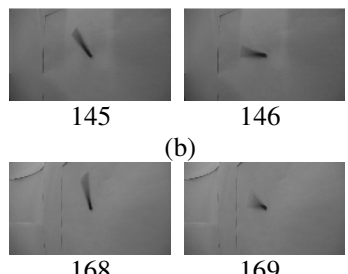

168

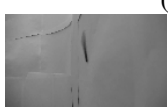

187

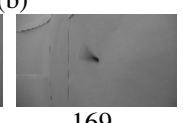

169

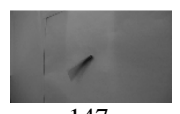

147

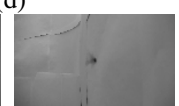

188

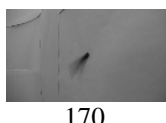

170

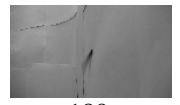

189

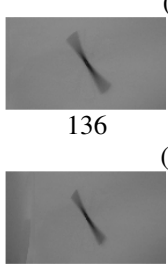

169

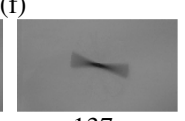

137

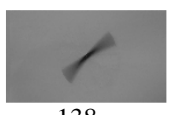

h)

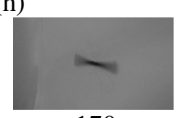

170

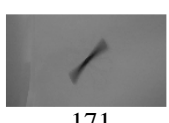

171

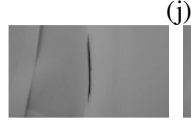

179

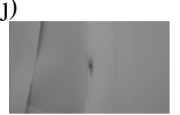

180

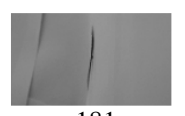

181

Fig. 16. Example frames from the sight axis deviation tests. Each video sequence is represented with four frames; the frame number is indicated under each image. (a) Half-bar: $\theta=15$ degrees. (b) Half-bar: $\theta=30$ degrees. (c) Half-bar: $\theta=45$ degrees. (d) Half-bar: $\theta=60$ degrees. (e) Half-bar: $\theta=75$ degrees. (f) Half-bar: $\theta=85$ degrees. (g) Bar: $\theta=15$ degrees. (h) Bar: $\theta=30$ degrees. (i) Bar: $\theta=45$ degrees. (j) Bar: $\theta=60$ degrees. (k) Bar: $\theta=75$ degrees. (l) Bar: $\theta=85$ degrees. Here $\theta$ is the sight axis deviation degree of the video camera.

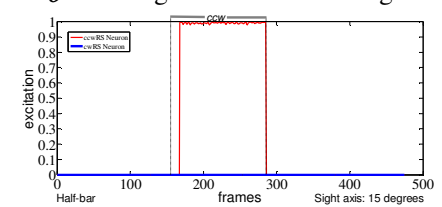

(a)

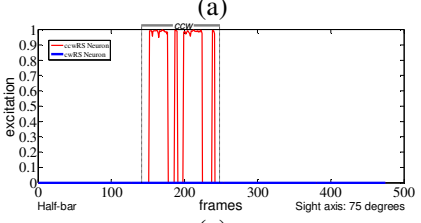

(e)

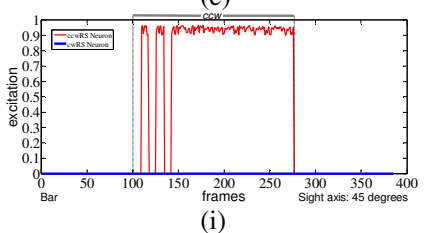

(i)

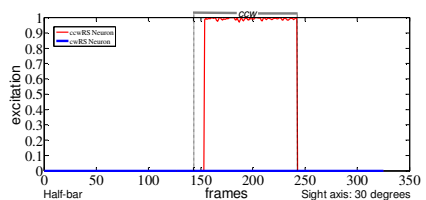

(b)

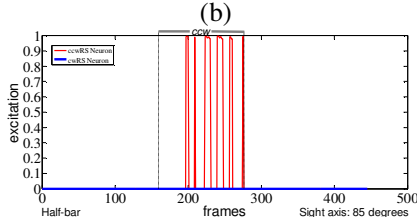

(f)

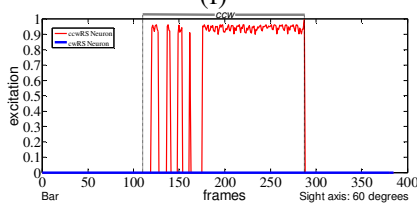

(j)

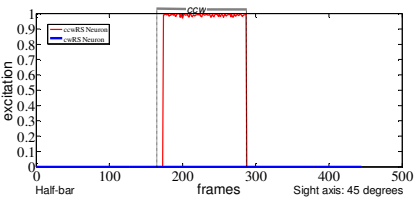

(c)

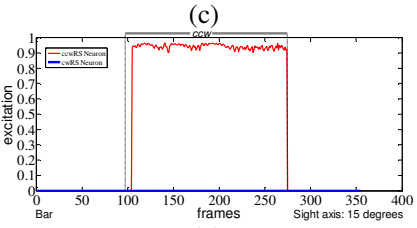

(g)

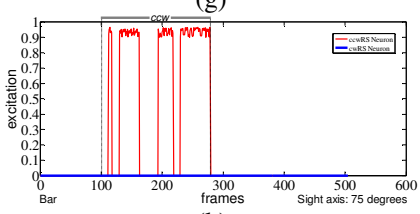

(k)

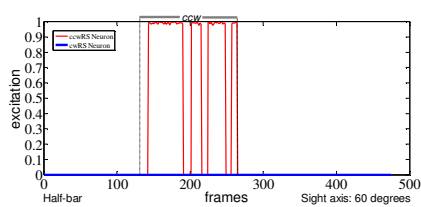

(d)

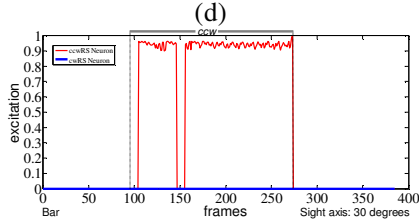

(h)

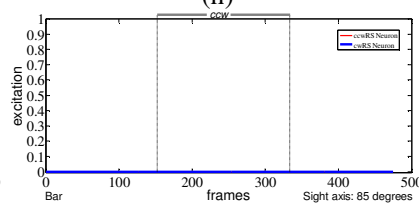

(l)

Fig. 17. Output curves of ccwRS neuron and cwRS neuron's excitation. Each sub-graph uniquely corresponds to the experimental result of the same identifier video sequence in Fig. 16.

video sequences, the angular velocity of the object's rotational motion is $15.7 \mathrm{rad} / \mathrm{s}$ (see Fig. 5).

In video sequence shown in Fig. 5 (a), a white block initially remains stationary from frame 1 to frame 92; it then rotates at a constant angular velocity in ccw from frame 93 to frame 219; finally remains stationary from frame 220 to the end. In video sequence shown in Fig. 5 (b), a white block remains stationary from frame 1 to frame 92, and rotates at a constant angular velocity in ccw from frame 93 to frame 217; then remains stationary in the remaining video frames. Similarly video sequence in Fig. 5 (c) and (d) simulates the $\mathrm{ccw}$ and $\mathrm{cw}$ rotational motion of a white half-bar; video sequence in Fig. 5

(e) and (f) simulate the ccw and cw rotational motion of a white bar respectively. The statistical results of these sequences are displayed in Table II.

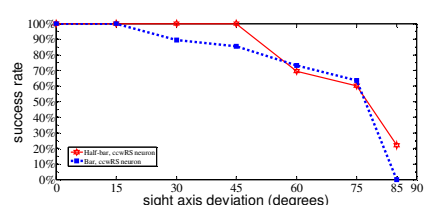

(a)

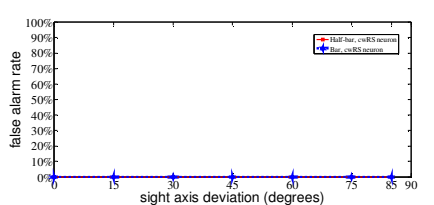

(b)

Fig. 18. CcwRS neuron's perception success rates and cwRS neuron's false alarm rates curves in the sight axis deviations tests. (a) The perception success rates of the ccwRS neuron. (b) The perception false alarm rates of cwRS neuron. 


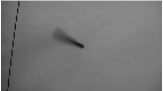

123

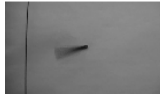

136

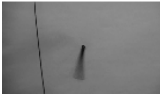

150

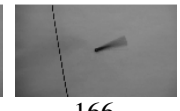

166

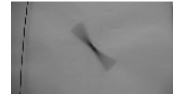

139

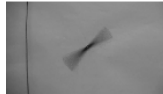

150

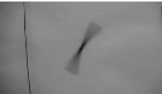

155

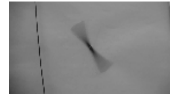

161

Fig. 19. Example frames of original video sequences from the varied intensity interference tests. Each original video sequence is represented with four frames; the frame number is indicated under each image. (a) A half-bar's ccw rotational motion mixed with interference. (b) A bar's ccw rotational motion mixed with interference.

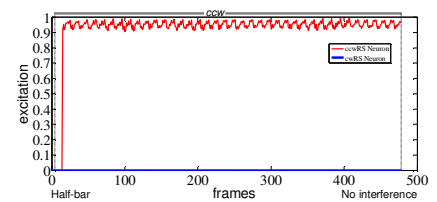

(a)

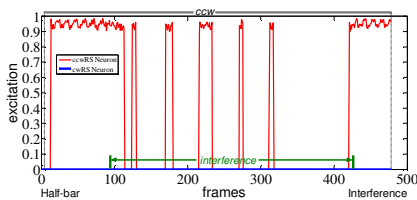

(b)

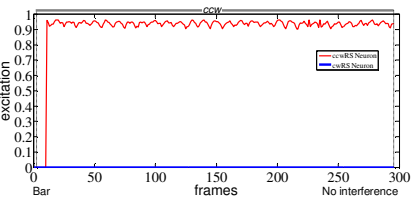

(c)

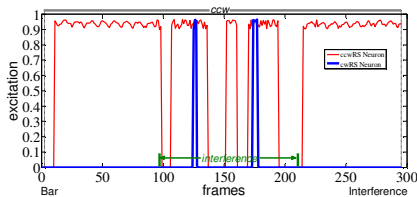

(d)

Fig. 20. Output curves of ccwRS neuron and cwRS neuron's excitation. (a) The experimental result of the video sequence in Fig. 19 (a), but the interference was fully erased (i.e. a half-bar's ccw rotational motion without interference.). (b) The test result of the video sequence in Fig. 19 (a). (c) The test result of the video sequence in Fig. 19 (b), but the interference was fully erased (i.e. a bar's ccw rotational motion without interference.). (b) The test result of the video sequence in Fig. 19 (b).

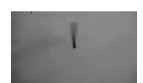

20

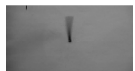

40

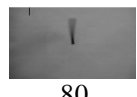

80

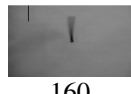

(a)
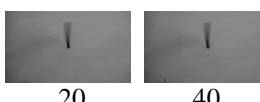

40
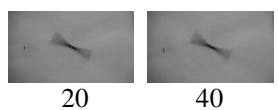

40

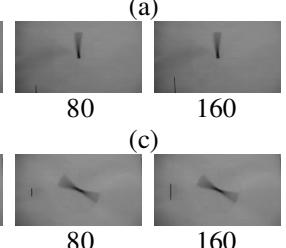

(e)

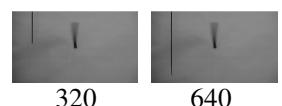

640

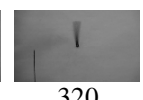

320
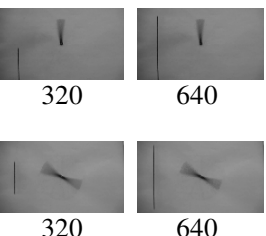

640

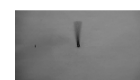

20

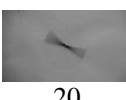

20

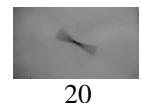

20

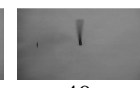

40

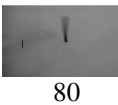

80

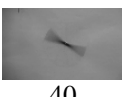

40

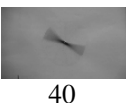

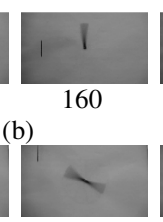

160

(d)

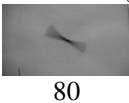

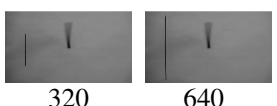

640
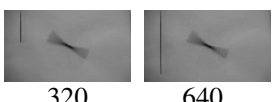

640

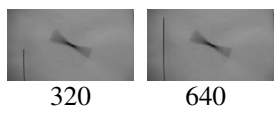

Fig. 21. Example frames from the varied intensity interference tests. By erasing different part (upper, middle and lower) of a cable, different intensity and swing amplitude interferences in different regions were formed. The resolution of each original video sequence is $720 \mathrm{P}$.The cable was partially erased and retained different height pixels in the three sections respectively, and forming eight types of interference of the different cable lengths: 0 pixels (the cable completely disappear, i.e. non-interference), 20 pixels, 40 pixels, 80 pixels, 160 pixels, 320 pixels, 640 pixels and 720 pixels (the full cable remains, see Fig. 19 (a), (b)). The length of interference (i.e. the retained cable length (pixels)) is indicated under each image. (a) Half-bar: interferences in the upper section. (b) Half-bar: interferences in the middle section. (c) Half-bar: interferences in the lower section. (d) Bar: interferences in the upper section. (e) Bar: interferences in the middle section. (f) Bar: interferences in the lower section.

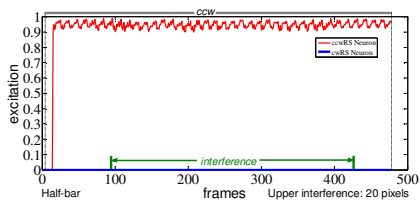

(a)

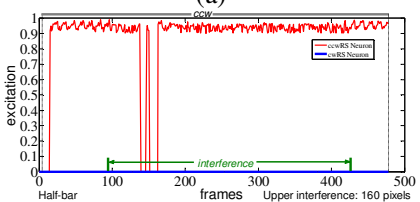

(d)

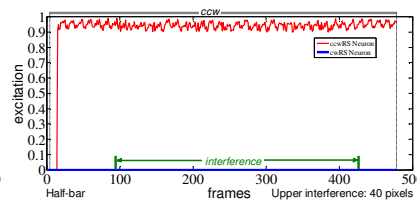

(b)

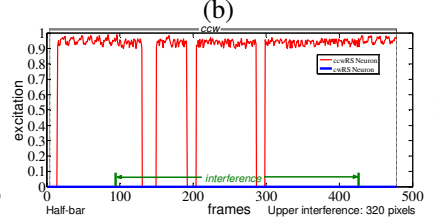

(e)

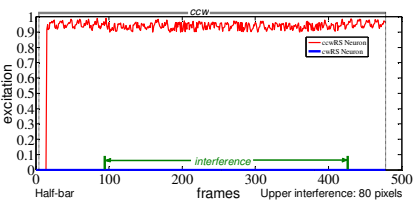

(c)

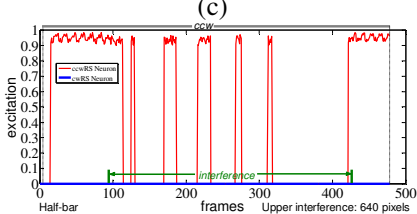

(f)

Fig. 22. Output curves of ccwRS neuron and cwRS neuron's excitation. Each sub-graph uniquely corresponds to the experimental results of a video sequence in Fig. 22 (a). (a) Half-bar: 20 pixels swing cable interference. (b) Half-bar: 40 pixels swing cable interference. (c) Half-bar: 80 pixels swing cable interference. (d) Half-bar: 160 pixels swing cable interference. (e) Half-bar: 320 pixels swing cable interference. (f) Half-bar: 640 pixels swing cable interference.

Fig. 6 shows the test outputs of the RMPNN against these simulated image sequences. Table II indicates that ccwRMPNN and cwRMPNN can correctly perceive the rotational motion for all simulated image sequences in these tests. From Fig. 6 and table II, we found that RMPNN worked very well for these simulated data sets.

\section{B. Real Scene Video Tests}

We continue to test the RMPNN with recorded video sequences from real or physical scenes - where the three objects, e.g., a black block, a black half-bar, and a black bar, were conducting $\mathrm{ccw}$ or $\mathrm{cw}$ rotational motion in a laboratory setting. Firstly, we recorded two video sequences in which a regular black bar $(60 \mathrm{~mm}$ in length, $5 \mathrm{~mm}$ in width) rotates around its center in ccw and cw respectively; then, we recorded a black half-bar (30 $\mathrm{mm}$ in length, $5 \mathrm{~mm}$ in width) rotates around its one fixed end in ccw and cw respectively. The rotational motion sequences of a block were edited from that of whole half-bar sequences, using video editing software (After Effects CS4, Adobe ${ }^{\circledR}$, USA). For each frame of the whole half-bar sequences, we erased almost the entire half-bar but leave its far end unchanged as the black block (see Fig. 7).

In video sequence shown in Fig. 7 (a) (561 frames), a black block remains stationary from frame 1 to frame 166; after that it rotates at variable angular velocities $(23.55 \sim 17.13 \mathrm{rad} / \mathrm{s})$ in ccw 
from frame 167 to frame 507; finally it remains stationary from frame 508 to the end. In video sequence Fig. 7 (b) (610 frames), a black block holds stationary from frame 1 to frame 170, and it rotates at an angular velocity $(12.99 \mathrm{rad} / \mathrm{s})$ in $\mathrm{cw}$ from frame 171 to frame 514, then keeps stationary from frame 515 to frame 610. In video sequences of Fig. 7(c) (d), the rotation pattern of a half-bar is the same as that of the block shown in Fig. 7(a) or Fig. 7 (b) respectively; In video sequences of Fig. 7(e) (f), the rotation pattern of a bar is similar to that of the block shown in Fig. 7(a) or Fig. 7 (b), except angular velocities: 23.55 18.84 rad/s in Fig. 7(e), and 16.38 15.7 rad/s in Fig. 7(f). The statistical results of these sequences are displayed in Table III.

As illustrated in Fig. 8, we can clearly see that the proposed
RMPNN works very well on these real scene video tests, although the rotation angular velocities of these objects are different.

\section{Boundary Condition Tests}

In the following tests, RMPNN will be challenged with several types of real scene video sequences under various conditions.

\section{1) Varied Inhibition Radius Tests}

In this sub-section, we examine the effect of inhibition radius on the performance of RMPNN. During our experiment, we found that the motion perception of RMPNN was influenced by different inhibition radius. Too small inhibition radius will cause the incorrect perception of motion cues; while too large

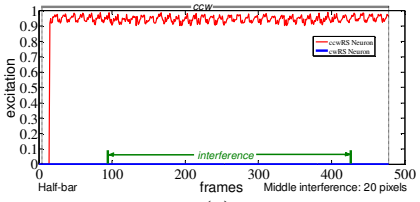

(a)

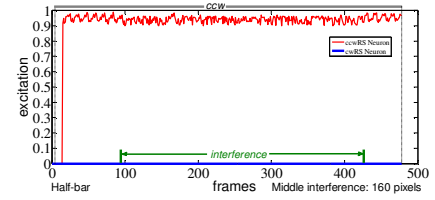

(d)

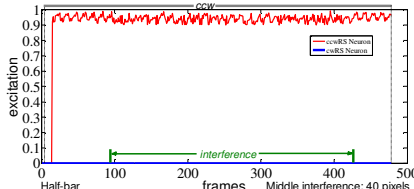

(b)

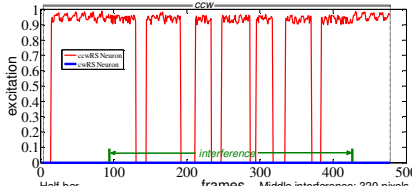

(e)

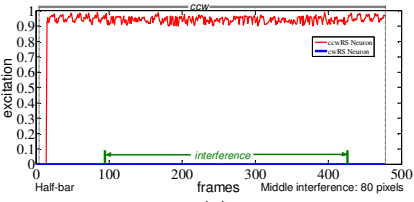

(c)

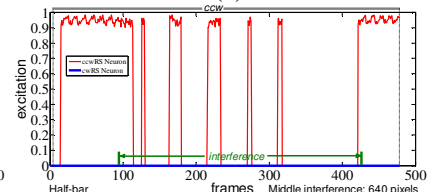

(f)

Fig. 23. Output curves of ccwRS neuron and cwRS neuron's excitation. Each sub-graph uniquely corresponds to the experimental results of a video sequence in Fig. 22 (b). (a) Half-bar: 20 pixels swing cable interference. (b) Half-bar: 40 pixels swing cable interference. (c) Half-bar: 80 pixels swing cable interference. (d) Half-bar: 160 pixels swing cable interference. (e) Half-bar: 320 pixels swing cable interference. (f) Half-bar: 640 pixels swing cable interference.

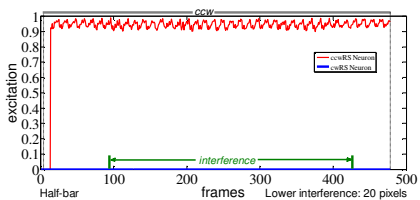

(a)

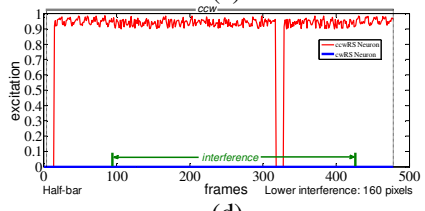

(d)

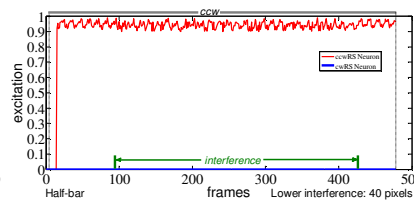

(b)

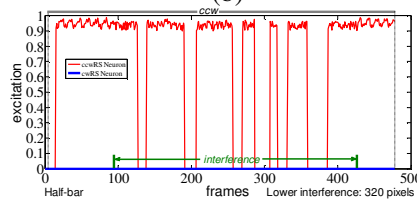

(e)

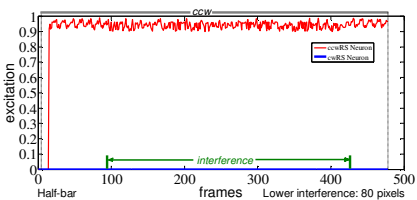

(c)

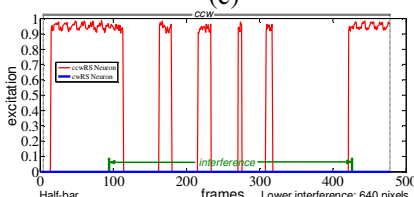

(f)

Fig. 24. Output curves of ccwRS neuron and cwRS neuron's excitation. Each sub-graph uniquely corresponds to the experimental results of a video sequence in Fig. 22 (c). (a) Half-bar: 20 pixels swing cable interference. (b) Half-bar: 40 pixels swing cable interference. (c) Half-bar: 80 pixels swing cable interference. (d) Half-bar: 160 pixels swing cable interference. (e) Half-bar: 320 pixels swing cable interference. (f) Half-bar: 640 pixels swing cable interference.

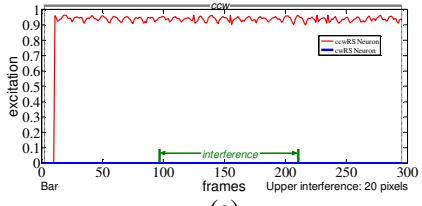

(a)

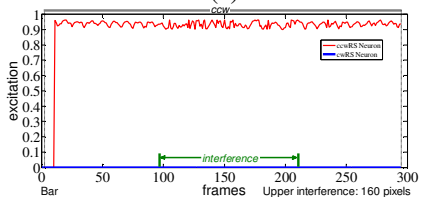

(d)

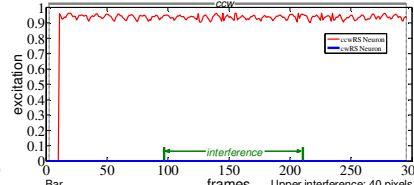

(b)

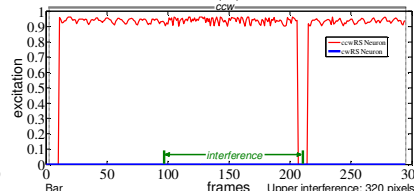

(e)

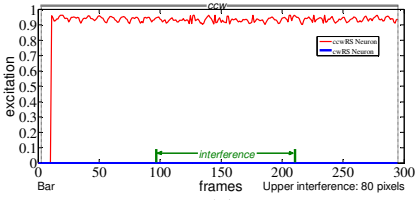

(c)

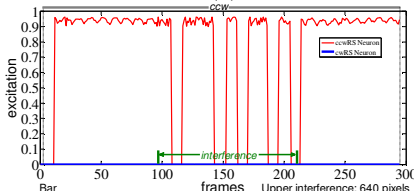

(f)

Fig. 25. Output curves of ccwRS neuron and cwRS neuron's excitation. Each sub-graph uniquely corresponds to the experimental results of a video sequence in Fig. 22 (d). (a) Bar: 20 pixels swing cable interference. (b) Bar: 40 pixels swing cable interference. (c) Bar: 80 pixels swing cable interference. (d) Bar: 160 pixels swing cable interference. (e) Bar: 320 pixels swing cable interference. (f) Bar: 640 pixels swing cable interference. 
inhibition radius will lead to superfluous computing during its visual information processing. To perceive effectively the motion cues, an appropriate inhibition radius value should be set in RMPNN.

Two recorded real scene videos were employed in this type of tests. One is the ccw rotational motion of a half-bar, and the other is the ccw rotational motion of a bar (see Fig. 9). In video sequence shown in Fig. 9 (a) (478 frames), a half-bar rotates at an angular velocity $(15.7 \mathrm{rad} / \mathrm{s})$ in ccw from frame 1 to the end. In video sequence shown in Fig. 9 (b) (296 frames), a bar rotates at an angular velocity $(20.93 \mathrm{rad} / \mathrm{s})$ in ccw from the first frame to the last frame. During the tests, all parameters are fixed except the inhibition radius values in the RMPNN. We set six different inhibition radius values in each video test respectively: $2,4,8,16,32$ and 64 pixels.

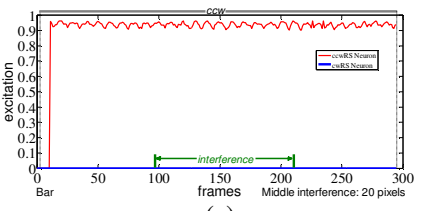

(a)

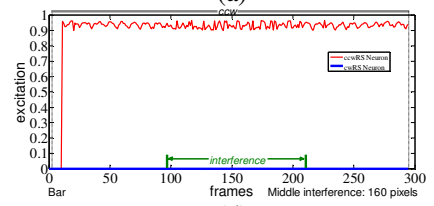

(d)

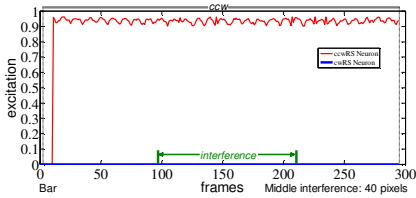

(b)

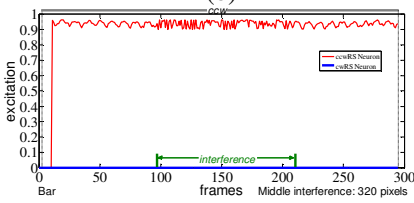

(e)

while the inhibition radius equaled to or greater than 32 pixels, both the ccwRS neuron and the cwRS neuron can correctly perceive the rotational motion of the bar. Subsequently we counted the perception success rate of the ccwRS neuron and the false alarm rate of the cwRS neuron in these different inhibition radius value tests, and plotted rate-inhibition radius curves, as shown in Fig. 11. From these results, we found that an appropriate inhibition radius affect the performance of the RMPNN in perceiving a rotational motion.

\section{2) Position Invariance and Varied Receptive Field Tests}

Position invariance is an important feature in the biological visual systems. Although an object in motion may appear at many different locations retinotopically, an animal can still perceive it correctly. This feature has been verified in

Fig. 26. Output curves of ccwRS neuron and cwRS neuron's excitation. Each sub-graph uniquely corresponds to the experimental results of a video sequence in Fig. 22 (e). (a) Bar: 20 pixels swing cable interference. (b) Bar: 40 pixels swing cable interference. (c) Bar: 80 pixels swing cable interference. (d) Bar: 160 pixels swing cable interference. (e) Bar: 320 pixels swing cable interference. (f) Bar: 640 pixels swing cable interference.

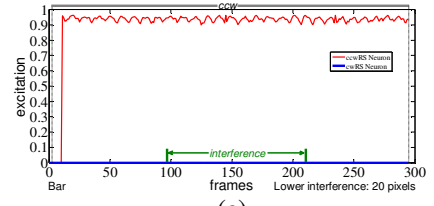

(a)

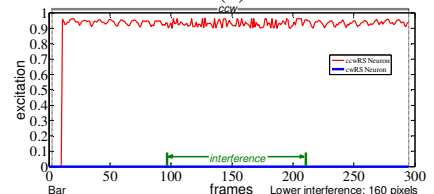

(d)

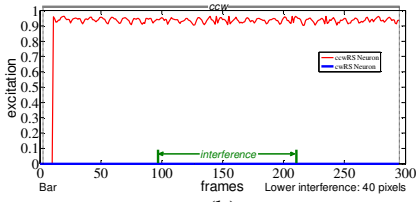

(b)

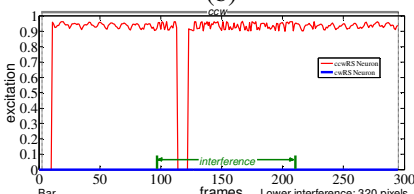

(e)

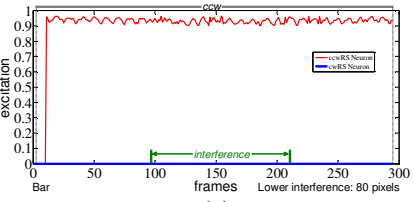

(c)

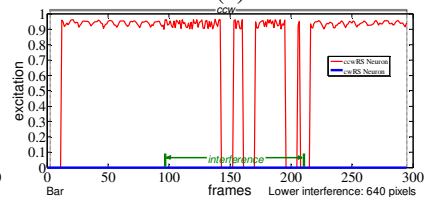

(f)

Fig. 27. Output curves of ccwRS neuron and cwRS neuron's excitation. Each sub-graph uniquely corresponds to the experimental results of a video sequence in Fig. 22 (f). (a) Bar: 20 pixels swing cable interference. (b) Bar: 40 pixels swing cable interference. (c) Bar: 80 pixels swing cable interference. (d) Bar: 160 pixels swing cable interference. (e) Bar: 320 pixels swing cable interference. (f) Bar: 640 pixels swing cable interference.

Fig. 10 shows the output excitation of the RMPNN in this set of tests. From Fig. 10 (a) (f), we can see that with the inhibition radius increasing, when the inhibition radius value equal to or greater than 4 pixels, both the ccwRS neuron and the cwRS neuron can correctly perceive the rotational motion of a half-bar. From Fig. $10(\mathrm{~g}) \sim(1)$, we can find that when the inhibition radius value is varied within $2 \sim 16$ pixels (Fig. $10(\mathrm{~g})$ $\sim(\mathrm{j})$ ), RMPNN cannot correctly perceive the rotational motion of a bar in ccw, and even appear some incorrect response as shown in Fig. 10 (i). With the growth of inhibition radius value,

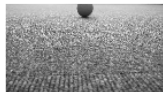

35

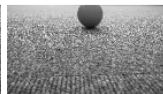

40

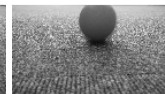

43

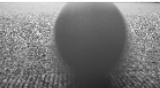

46

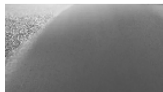

48

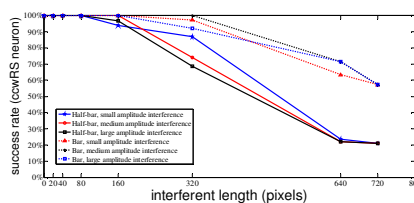

(a)

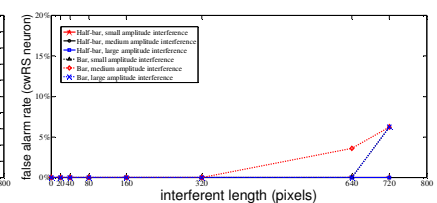

(b)

Fig. 28. CcwRS neuron's perception success rates and cwRS neuron's false alarm rates curves in the varied intensity interference tests. (a) The perception success rates of ccwRS neuron. (b) The perception false alarm rate of cwRS neuron.

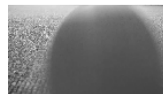

1

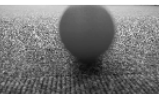

3

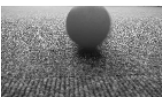

5

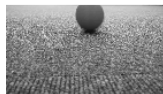

8

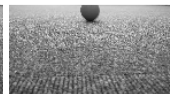

12

Fig. 29. Example frames from the non-rotational motion tests. Each video sequence is represented with five frames; the frame number is indicated under each image. (a) A ball is approaching to the video camera. (b) A ball is receding from the video camera. 
neurophysiological experiments, e.g. Sakata et al. found that the receptive fields of RS neurons in the cerebral cortex of the monkey are quite large, and a shift of the center of rotation within the receptive field do not cause a large difference in response [43].

In order to test the response of RMPNN to the rotational motion in different regions of the field of view, a set of experiments were designed. We recorded the rotational motion of a half-bar and a bar in ccw at different regions of the field of view respectively: top-left, bottom-left, top-right and bottom-right (see Fig. 12). In these video sequences, the rotational motion patterns in ccw of the half-bar and the bar are similar to that of the previous simulated visual stimuli generated by a computer except for the difference of the rotation angular velocities (19.83 23.55 rad/s in this type of tests). In each video sequence, the object initially remains stationary several seconds, and then rotates in ccw for a few of seconds, finally stops rotating and remains stationary until the end of the video sequence. Then the RMPNN will be challenged by these video sequences.

Experimental results are shown in Fig. 13 and Fig. 14. The two charts show that RMPNN can correctly perceive the rotational motion of a half-bar and a bar, and regardless of their different position in the field of view. The results show that the proposed RMPNN is similar to the biological vision neural network - it also has the position invariance feature, which is compatible with the vector field hypothesis [11].

\section{3) Sight Axis Deviation Tests}

In the above tests, the sight axis of video camera is perpendicular to the rotating plane of object, and RMPNN responded well to these rotational motions of objects. However, rotation motion may happen in different planes. To test the impact of the sight axis deviation on the performance of RMPNN, we will use video sequences with different camera sight axis deviated on the horizontal plane (Fig. 15).

We progressively increased the horizontal deviation angle (on the X-Z horizontal plane, see Fig. 15) between the sight axis and the vertical line on the fronto-parallel plane. In the adjacent video sequences of these tests, the camera's sight axis changed from the vertical line by 15 degrees each time until approached parallel lines of the fronto-parallel plane. As the sight axis deviation reached over 75 degrees, we continued to increase 10 deg to make it at 85 degrees for the final video sequences. The schematic diagram of sight axis deviations tests is shown in Fig. 15 , and example frames of each video sequences are shown in Fig. 16.

The rotational motion patterns in ccw of a half-bar and a bar also are similar to that of the previous simulated visual stimuli generated by a computer except for the difference of the rotation angular velocities (13.46 23.55 rad/s in this type of tests). Fig. 17 and 18 show the experimental results in these tests. From these experimental results we can see that when the sight axis deviation angle is small up to 30 degrees, RMPNN can correctly perceive the rotational motion of a half-bar and a bar. This suggests that the RMPNN is robust to small perturbation in terms of rotational planes. However, with the sight axis deviation angle increasing heavily (60 deg in the video sequence shown in Fig. 16 (d), and 45 deg in the video sequence shown in Fig. 16 (i)), the perception success rate of the ccwRS neuron began to decline. When the sight axis is almost parallel to the fronto-parallel plane, the perception success rate of ccwRS neuron reduced to the minimum, and the false alarm rate of cwRS neuron reached to the maximum (see Fig. 18).

\section{4) Varied Intensity Interference Tests}

In order to test the interference influence on RMPNN, a set of video sequences about varied intensity interference scenes were created with a swing black headphone cable. We recorded two original video sequences with a half-bar and a bar rotating in $\mathrm{ccw}$ from the first frame to the end respectively. In the intermediate time section of each video sequence, a vertical black headphone cable is located on the left side of the rotating object, as interference in the field of view, simulating a pendulum swinging to affect the rotational motion perception of the RMPNN (see Fig. 19). In each of the two video sequences, the cable initially remains stationary for a few

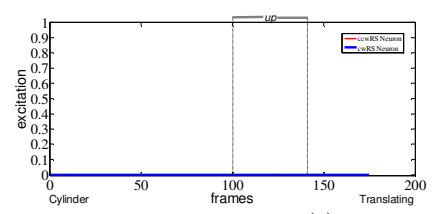

(a)

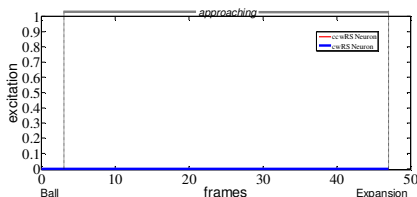

(b)

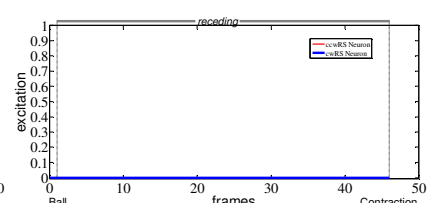

(c)

Fig. 30. Output curves of ccwRS neuron and cwRS neuron's excitation in the non-rotational motion tests. Each sub-graph uniquely corresponds to the experimental results of a video sequence in these tests. (a) The experimental results corresponding to the video sequences shown in Fig.2 (a). (b) The experimental results corresponding to the video sequences shown in Fig.29 (a). (c) The experimental results corresponding to the video sequences shown in Fig.29 (b).
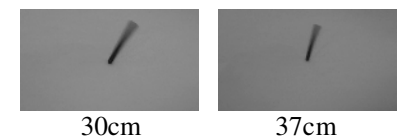

$37 \mathrm{~cm}$

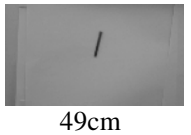

(a)

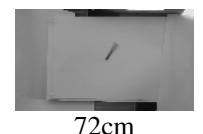

$72 \mathrm{~cm}$

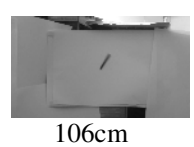

$106 \mathrm{~cm}$

Fig. 31. Varied object distances tests. (a) Example frames from the different object distances tests. Each video sequence is represented with one frame; the object distance is indicated under each image. (b) The perception success rates of ccwRS neuron and the false alarm rates of cwRS neuron's curves in the varied object distances tests. 
seconds, and then swings around for several seconds before rests. We then use After Effects CS4 to erase the whole cable to obtain two non-interference rotational motion video sequences of a half-bar and a bar respectively. Similarly, we get different intensity and swing amplitude interferences in different regions by erasing different part (upper, middle and lower) of the cable, as shown in Fig. 21.

The resolution of each original video sequence is $720 \mathrm{P}$. The cable was partially erased and retained different height pixels in the three sections respectively, and forming eight types of interference of the different cable lengths: 0 pixels (the cable completely disappear, i.e. non-interference), 20 pixels, 40 pixels, 80 pixels, 160 pixels, 320 pixels, 640 pixels and 720 pixels (the full cable remains), see Fig. 19 and 21.

In these video sequences, all rotational motion patterns of the half-bar and the bar are similar except for the different lengths of the cable interference. In the half-bar rotation video sequences, each video sequence has 478 frames. A half-bar rotates in ccw at an angular velocity $(15.7 \mathrm{rad} / \mathrm{s})$ throughout the whole video sequence. The cable keeps stationary state from frame 1 to frame 94; and then it simulates the pendulum swinging motion from frame 95 to frame 426; after that it stops swinging and holds still from frame 427 to frame 478. Similarly, each video sequence of a bar rotation has 296 frames. A bar rotates in ccw at an angular velocity $(20.93 \mathrm{rad} / \mathrm{s})$ throughout the whole video sequence. The cable keeps stationary state from frame 1 to frame 97; and then it simulates the pendulum swinging around motion from frame 98 to frame 209; after that it stops swinging and holds still from frame 210 to the last frame.

The performance of the RMPNN against two test results, i.e. the 0 pixels interference (i.e. the cable completely disappear) and the 720 pixels interference (i.e. the full cable remains), are shown in Fig. 20. The other test results of the remaining various intensity interferences video sequences are shown in Fig. 22, 23, $24,25,26$ and 27 respectively. We also counted the perception success rates of the ccwRS neuron and the false alarm rates of the cwRS neuron in this type of experiments, and plotted the rate-interferent curves, as shown in Fig. 28. From these tests results, we can find that the proposed RMPNN is robust, as small intensity of interference did not affect its performance. However, its performance will decrease as the strength of the interference increases.

\section{5) Non-rotational Motion Tests}

To see the selectiveness of the RMPNN, we will challenge it with three different types of non-rotational motion video sequences. One type is upward translation, the second one is expansion motion and the third is contraction motion. In Fig.2 (a), a white cylinder is moving upward; In Fig. 29 (a), a ball is approaching to the camera; In Fig. 29 (b), a ball is receding from the camera.

Tests' results are shown in Fig. 30. As shown in these figures, RMPNN has no response to these non-rotational motions, showing excellent selectiveness of the proposed rotational selective neural network.

\section{Discussions}

In the above sections, the presented RMPNN has been tested using several types of rotational motion video sequences under various conditions. All of these experiments have demonstrated that the RMPNN has a reliable ability to perceive rotational motion. Experimental results showed that the properties of the RMPNN are coincide with the most of main functional properties of rotation selective neurons in monocular viewing condition [6], [43], [44], including rotational motion selective, rotation direction selective, response delay, position invariant, and no-preference for non-rotational basic motion (translation, expansion, and contraction).

However, the proposed RMPNN will not respond to the rotational motion in the sagittal plane [43]. The RMPNN is a monocular visual system and it cannot deal with rotating object in sagittal plane. This is because, the projection of a rotating object, e.g. a bar in the sagittal plane, on to the field of view, will form a periodically expanding/contracting line in a monocular visual system. A binocular vision system with two RMPNN, for example, may still be able to recognize the rotating object, even if a bar may be in the sagittal plane to one of the RMPNN, given the distances between the two RMPNN is big enough to allow the other RMPNN having adequate angle to perceive the rotation (for example, Fig. 16 18).

The proposed RMPNN contains only sixteen directional selective neurons. With this low spatial angular resolution (about 22.5 degrees each and 16 DSNNs to cover the full circle), it can only cope with rotational motion within a certain range of angular velocities. This means that the RMPNN can perceive rotational motion at appropriate rotation speeds effectively but may not respond well to those with too slowly or too fast angular velocities.

Neurophysiological study showed that rotation selective neurons can be classified into three categories according to their responding behaviours to the size of rotational objects [43]. Sakata et. al. experiments showed that most of these types of rotation selective neurons' responses increase with the increase of object size, though each type with different size preference [43]. We examined the influence of object size on the performance of the RMPNN by placing the object with varied distance before the camera. Five separate tests were conducted with distance at $30,37,49,72$, and $106 \mathrm{~cm}$ respectively (see Fig. 31 (a)). From these sampled video sequences, we can see a half-bar in the field of view is getting smaller and smaller as the object distance increasing. As shown in Fig. 31 (b), within a certain range, the changes of object distance (i.e., object size) stimulated similar performance pattern compare to Sakata et. al. (1994) to the RMPNN - the perception success rate increases with the increase of objects size (i.e., closer distance). As detailed in sections III and demonstrated with experiments (Fig.9 11), the inhibition radius predefined in a RMPNN can affect its preference to different object size. This suggests that a population of RMPNNs with different inhibition radius could exhibit variety of object size preferences for complex visual scenarios.

In our experiments, the original data feed to the RMPNN are the frames extracted from video sequences. Hence, the video 
quality (e.g. video definition, data rate, frame rate and so on) has a significant influence on the performance of the RMPNN if in a real time application. The key of video technology is video encoding, which directly affects the level of video quality. We hope that in the future work, High Efficiency Video Coding (HEVC) can be introduced to the real time data acquisition loop of the visual information processing system, to effectively improve the efficiency of video encoding, to reduce the corresponding computational complexity [64]-[67] and to enhance the performance of the RMPNN.

\section{CONCLUSION}

In the above sections, we proposed a new rotational selective visual neural network, RMPNN, with a specialized spatiotemporal information processing mechanism based on asymmetrically inhibited directional selective neuron models. The directional selective neurons are arranged in a specific order similar to that of the directional columns in the cerebral cortex for perceiving the specific rotational motion cues. Systematic experiments demonstrated that the performance of the proposed RMPNN is robust against position invariance, sight axis deviation, certain range of rotation velocities, various interferences and objects size. These characters are in consistence with those features of rotation selective neurons revealed in neurophysiological studies [5], [6], [8], [10], [11], [21], [43]. The RMPNN has also demonstrated robust rotation selectiveness of the RMPNN when challenged with non-rotational principle motion patterns (e.g, translation, expansion, and contraction) in our experiments. As the first bio-plausible computational model for rotational motion perception, this research is a significant step towards deep understanding of dynamic visual information processing in both biological and artificial vision systems.

In the future, we may expand our investigation of the perception of rotational motion in 3D space with binocular RMPNN. The RMPNN may also combine with other types of neurons, to perceive complex motion patterns in real world.

\section{ACKNOWLEDGMENT}

The authors would like to thank the anonymous reviewers for their valuable comments and thank the editors of this paper for their kind support. The authors also thank Mr. Cheng Hu for his help in video recording for the experiments.

\section{References}

[1] J. J. Koenderink and A. J. van Doorn, "Local structure of movement parallax of the plane," J. Opt. Soc. Am., vol. 66, no. 7, pp. 717-723, 1976.

[2] A. Verri, F. Girosi, and V. Torre, "Differential techniques for optical flow," J. Opt. Soc. Am. A, vol. 7, no. 5, pp. 912-922, 1990.

[3] D. H. Hubel and T. N. Wiesel, "Receptive fields, binocular interaction and functional architecture in the cat's visual cortex," J. Physiol., vol. 160, no. 1, pp. 106-154, 1962.

[4] J. H. Maunsell and D. C. Van Essen, "Functional properties of neurons in middle temporal visual area of the macaque monkey. I. Selectivity for stimulus direction, speed, and orientation," $J$. Neurophysiol., vol. 49, no. 5, pp. 1127-1147, 1983.

[5] H. Sakata, H. Shibutani, K. Kawano, and T. L. Harrington, "Neural mechanisms of space vision in the parietal association cortex of the monkey," Vision Res., vol. 25, no. 3, pp. 453-463, 1985 .

[6] H. Sakata, H. Shibutani, Y. Ito, and K. Tsurugai, "Parietal cortical neurons responding to rotary movement of visual stimulus in space," Exp. brain Res., vol. 61, no. 3, pp. 658-663, 1986.

[7] K. Tanaka, K. Hikosaka, H. Saito, M. Yukie, Y. Fukada, and E. Iwai, "Analysis of local and wide-field movements in the superior temporal visual areas of the macaque monkey," J. Neurosci., vol. 6, no. 1, pp. 134-144, 1986.

[8] K. Tanaka and H. Saito, "Analysis of motion of the visual field by direction, expansion/contraction, and rotation cells clustered in the dorsal part of the medial superior temporal area of the macaque monkey," J. Neurophysiol., vol. 62, no. 3, pp. 626-641, 1989.

[9] R. H. Wurtz, D. S. Yamasaki, C. J. Duffy, and J. P. Roy, "Functional specialization for visual motion processing in primate cerebral cortex.," Cold Spring Harb. Symp. Quant. Biol., vol. 55, pp. 717-727, 1990.

[10] C. J. Duffy and R. H. Wurtz, "Sensitivity of MST neurons to optic flow stimuli. I. A continuum of response selectivity to large-field stimuli," J. Neurophysiol., vol. 65, no. 6, pp. 1329-1345, 1991.

[11] C. J. Duffy and R. H. Wurtz, "Sensitivity of MST neurons to optic flow stimuli. II. Mechanisms of response selectivity revealed by small-field stimuli," J. Neurophysiol., vol. 65, no. 6, pp. 1346-1359, 1991.

[12] M. S. Graziano, R. a Andersen, and R. J. Snowden, "Tuning of MST neurons to spiral motions," J. Neurosci., vol. 14, no. 1, pp. 54-67, 1994.

[13]L. Lagae, H. Maes, S. Raiguel, D. K. Xiao, and G. a Orban, "Responses of macaque STS neurons to optic flow components: a comparison of areas MT and MST," J. Neurophysiol., vol. 71, no. 5, pp. 1597-1626, 1994.

[14] R. D. Santer, P. J. Simmons, and F. C. Rind, "Gliding behaviour elicited by lateral looming stimuli in flying locusts," J. Comp. Physiol. A, vol. 191, no. 1, pp. 61-73, 2005.

[15]R. D. Santer, Y. Yamawaki, F. C. Rind, and P. J. Simmons, "Motor activity and trajectory control during escape jumping in the locust Locusta migratoria," J. Comp. Physiol. A, vol. 191, no. 10, pp. 965-975, 2005.

[16] A. D. Huberman, W. Wei, J. Elstrott, B. K. Stafford, M. B. Feller, and B. a. Barres, "Genetic Identification of an On-Off Direction-Selective Retinal Ganglion Cell Subtype Reveals a Layer-Specific Subcortical Map of Posterior Motion," Neuron, vol. 62 , no. 3, pp. 327-334, 2009.

[17]H. Fotowat and F. Gabbiani, "Collision Detection as a Model for Sensory-Motor Integration,” Annu. Rev. Neurosci., vol. 34, no. 1 , pp. 1-19, 2011.

[18] A. Cruz-Martín, R. N. El-Danaf, F. Osakada, B. Sriram, O. S Dhande, P. L. Nguyen, E. M. Callaway, A. Ghosh, and A. D. Huberman, "A dedicated circuit links direction-selective retinal ganglion cells to the primary visual cortex," Nature, vol. 507, no 7492, pp. 358-361, 2014.

[19]L. Leinonen, "Functional properties of neurones in the posterior part of area 7 in awake monkey.," Acta Physiol. Scand., vol. 108, no. 3, pp. 301-308, 1980.

[20] G. Rizzolatti, G. Rizzolatti, C. Scandolara, C. Scandolara, M. Matelli, M. Matelli, M. Gentilucci, and M. Gentilucci, “Afferent properties of periarcuate neurons in macaque monkeys. II. Visua responses," Behav. Brain Res., vol. 2, no. 2, pp. 147-163, 1981.

[21] H. Saito, M. Yukie, K. Tanaka, K. Hikosaka, Y. Fukada, and E. Iwai, "Integration of direction signals of image motion in the superior temporal sulcus of the macaque monkey," J. Neurosci., vol. 6 , no. 1 , pp. $145-157,1986$.

[22] P. Cavanagh and O. E. Favreau, "Motion aftereffect: A global mechanism for the perception of rotation," Perception, vol. 9, no. 2. pp. 175-182, 1980.

[23] J. T. Petersik, A. Shepard, and R. Malsch, "A three-dimensional motion aftereffect produced by prolonged adaptation to a rotation simulation," Perception, vol. 13, no. 4, pp. 489-497, 1984.

[24] M. Hershenson, "Visual system responds to rotational and size-change components of complex proximal motion patterns," Percept. Psychophys., vol. 42, no. 1, pp. 60-64, 1987.

[25] M. B. Wall, A. Lingnau, H. Ashida, and A. T. Smith, "Selective visual responses to expansion and rotation in the human MT complex revealed by functional magnetic resonance imaging 
adaptation," Eur. J. Neurosci., vol. 27, no. 10, pp. 2747-2757, 2008.

[26] M. C. Morrone, M. Tosetti, D. Montanaro, A. Fiorentini, G. Cioni, and D. C. Burr, "A cortical area that responds specifically to optic flow, revealed by fMRI," Nat. Neurosci., vol. 3, no. 12, pp. 1322-1328, 2000

[27] A. Koban and R. Cook, "Rotational object discrimination by pigeons," J. Exp. Psychol. Anim. Behav. Process., vol. 35, no. 2, pp. 250-265, 2009.

[28] J. F. Nankoo, C. R. Madan, M. L. Spetch, and D. R. Wylie, "Perception of complex motion in humans and pigeons (Columba livia)," Exp. Brain Res., vol. 232, pp. 1843-1853, 2014.

[29] J. a. Marshall, "Self-organizing neural networks for perception of visual motion," Neural Networks, vol. 3, no. 1, pp. 45-74, 1990.

[30]F. C. Rind and D. I. Bramwell, "Neural network based on the input organization of an identified neuron signaling impending collision," J. Neurophysiol., vol. 75, no. 3, pp. 967-985, 1996.

[31] T. Tversky and R. Miikkulainen, "Modeling directional selectivity using self-organizing delay-adaptation maps," Neurocomputing, vol. 44-46, no. June, pp. 679-684, 2002.

[32] S. Yue and F. C. Rind, "Collision detection in complex dynamic scenes using an LGMD-based visual neural network with feature enhancement," IEEE Trans. Neural Netw., vol. 17, no. 3, pp. 705-716, 2006.

[33]Z. Zhang, S. Yue, and G. Zhang, "Fly visual system inspired artificial neural network for collision detection," Neurocomputing, vol. 153, pp. 221-234, 2015.

[34] G. A. Orban, L. Lagae, A. Verri, S. Raiguel, D. Xiao, H. Maes, and V. Torre, "First-order analysis of optical flow in monkey brain," Proc. Natl. Acad. Sci. U. S. A., vol. 89, no. April, pp. 2595-2599, 1992.

[35]I. K. King, J.-S. Liaw, and M. a. Arbib, "A neural network for the detection of rotational motion," in IJCNN-91-Seattle International Joint Conference on Neural Networks, 1991, vol. i, pp. 707-712.

[36] A. Guo, H. Sun, and X. Yang, “A multilayer neural network model for perception of rotational motion," Sci. China. Ser. C, vol. 40, no. 1, pp. 90-100, 1997.

[37] M. Sereno and M. Sereno, "Learning to see rotation and dilation with a Hebb rule," Advances in Neural Information Processing Systems 3. pp. 320-326, 1991.

[38] K. Zhang, M. Sereno, and M. Sereno, "Emergence of Position-Independent Detectors of Sense of Rotation and Dilation With Hebbian Learning - an Analysis," Neural Comput., vol. 5, pp. 597-612, 1993.

[39] S. a. Beardsley and L. M. Vaina, "Computational modeling of optic flow selectivity in MSTD neurons," Netw. Comput. Neural Syst., vol. 9, no. 4, pp. 467-493, 1998.

[40] S. a. Beardsley, R. L. Ward, and L. M. Vaina, "A neural network model of spiral-planar motion tuning in MSTd," Vision Res., vol. 43 , no. 5, pp. 577-595, 2003.

[41]E. T. Rolls and S. M. Stringer, "Invariant global motion recognition in the dorsal visual system: a unifying theory," Neural Comput., vol. 19, no. 1, pp. 139-169, 2007.

[42] W. Yang, L. Zhang, and L. Ma, "Computational model for rotation-invariant perception," Proc. - Third Int. Conf. Nat. Comput. ICNC 2007, vol. 2, pp. 144-148, 2007.

[43] H. Sakata, H. Shibutani, Y. Ito, K. Tsurugai, S. Mine, and M. Kusunoki, "Functional properties of rotation-sensitive neurons in the posterior parietal association cortex of the monkey.," Exp. Brain Res., vol. 101, no. 2, pp. 183-202, 1994

[44] K. M. Sakata H, Taira M, "The TINS Lecture The parietal association cortex in depth perception and visual control of hand action[J]. Trends in neurosciences," Trends Neurosci., vol. 20, no. 8, pp. $350-357,1997$.

[45] G. P. Caplovitz and P. U. Tse, "V3A processes contour curvature as a trackable feature for the perception of rotational motion," Cereb. Cortex, vol. 17, no. 5, pp. 1179-1189, 2007.

[46]B. Hassenstein and W. Reichardt, "Systemtheoretische Analyse der Zeit-, Reihenfolgen- und Vorzeichenauswertung bei der Bewegungsperzeption des Rüsselkäfers Chlorophanus," Zeitschrift für Naturforsch. B, vol. 11, no. 9, pp. 513-524, 1956.

[47] H. B. Barlow and W. R. Levick, "The mechanism of directionally selective units in rabbit's retina," J. Physiol., vol. 178, no. 3, pp. 477-504, 1965.
[48]N. Franceschini, a Riehle, and A. Le Nestour, Directionally selective motion detection by insect neurons. 1989.

[49]F. C. Rind, “A Directionally Selective Motion-Detecting Neurone in the Brain of the Locust: Physiological and Morphological Characterization," J. Exp. Biol., vol. 149, pp. 1-19, 1990.

[50]F. C. Rind, "Identification of directionally selective motion-detecting neurones in the locust lobula and their synaptic connections with an identified descending neurone," J. Exp. Biol. vol. 149, pp. 21-43, 1990

[51] A. Borst and J. Haag, "Neural networks in the cockpit of the fly," J. Comp. Physiol. A, vol. 188, no. 6, pp. 419-437, 2002.

[52] S. I. Fried, T. a Münch, and F. S. Werblin, "Mechanisms and circuitry underlying directional selectivity in the retina.," Nature, vol. 420, no. 6914, pp. 411-414, 2002.

[53] N. J. Priebe and D. Ferster, "Direction selectivity of excitation and inhibition in simple cells of the cat primary visual cortex," Neuron, vol. 45, no. 1, pp. 133-145, 2005.

[54]R. Kanjhan and B. Sivyer, "Two types of ON direction-selective ganglion cells in rabbit retina," Neurosci. Lett., vol. 483, no. 2, pp. 105-109, 2010.

[55] J. P. Gabriel, C. a. Trivedi, C. M. Maurer, S. Ryu, and J. H. Bollmann, "Layer-Specific Targeting of Direction-Selective Neurons in the Zebrafish Optic Tectum," Neuron, vol. 76, no. 6 , pp. 1147-1160, 2012.

[56] W. E. Reichardt and R. W. Schlögl, "A two dimensional field theory for motion computation - First order approximation; translatory motion of rigid patterns," Biol. Cybern., vol. 60, no. 1, pp. 23-35, 1988.

[57]S. Yue and F. Claire Rind, "Visual motion pattern extraction and fusion for collision detection in complex dynamic scenes," Comput. Vis. Image Underst., vol. 104, no. 1, pp. 48-60, 2006.

[58] S. Yue and F. C. Rind, "A synthetic vision system using directionally selective motion detectors to recognize collision," Artif. Life, vol. 13, no. 2, pp. 93-122, 2007.

[59] S. Yue and F. C. Rind, "Postsynaptic organisations of directional selective visual neural networks for collision detection," Neurocomputing, vol. 103, pp. 50-62, 2013.

[60] S. Yue and F. C. Rind, "Redundant neural vision systems-competing for collision recognition roles," IEEE Trans. Auton. Ment. Dev., vol. 5, no. 2, pp. 173-186, 2013.

[61] T. D. Albright, R. Desimone, and C. G. Gross, "Columnar organization of directionally selective cells in visual area MT of the macaque," J. Neurophysiol., vol. 51, no. 1, pp. 16-31, 1984.

[62] M. C. Morrone, D. C. Burr, and L. M. Vaina, "Two stages of visual processing for radial and circular motion," Nature, vol. 376, no. 6540, pp. 507-509, 1995.

[63] S. Yue, F. C. Rind, M. S. Keil, J. Cuadri, and R. Stafford, “A bio-inspired visual collision detection mechanism for cars: Optimisation of a model of a locust neuron to a novel environment," Neurocomputing, vol. 69, no. 13-15, pp. 1591-1598, 2006

[64] C. Yan, Y. Zhang, J. Xu, F. Dai, L. Li, Q. Dai, and F. Wu, “A highly parallel framework for HEVC Coding unit partitioning tree decision on many-core processors," IEEE Signal Process. Lett., vol. 21, no. 5, pp. 573-576, 2014.

[65]C. Yan, Y. Zhang, J. Xu, F. Dai, J. Zhang, Q. Dai, and F. Wu, "Efficient parallel framework for HEVC motion estimation on many-core processors," IEEE Trans. Circuits Syst. Video Technol., vol. 24, no. 12, pp. 2077-2089, 2014.

[66]C. Yan, Y. Zhang, F. Dai, J. Zhang, L. Li, and Q. Dai, "Efficient parallel HEVC intra-prediction on many-core processor," Electron. Lett., vol. 50, no. 11, pp. 805-806, 2014.

[67] C. Yan, Y. Zhang, F. Dai, X. Wang, L. Li, and Q. Dai, "Parallel deblocking filter for HEVC on many-core processor," Electron. Lett., vol. 50, no. 5, pp. 367-368, 2014. 


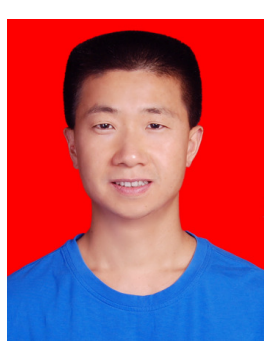

Bin Hu received the B.Eng. degree from Central South University, Changsha, China, in 2000, and the M.Eng degree from Guizhou University, Guiyang, China, in 2007, where he is currently pursuing the $\mathrm{Ph} . \mathrm{D}$. degree with the College of Computer Science and Technology.

$\mathrm{He}$ is an Associate Professor of the College of Computer Science and Technology, Guizhou University, China. He also works as a Research Assistant on biological visual neural network for Computational Intelligence Laboratory at the University of Lincoln, UK. His current research interests include biological visual neural systems, computer vision, artificial intelligence, and software engineering.

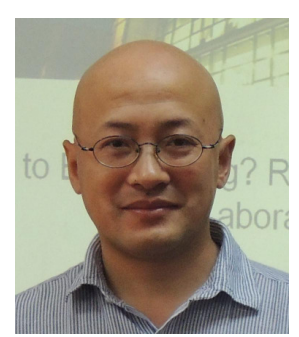

Shigang Yue (M'05) received Ph.D and MSc degree from Beijing University of Technology (BJUT), Beijing, China, in 1996 and 1993 respectively, BEng. degree from Qingdao University of Technology, China, in 1988.

$\mathrm{He}$ is a Professor in the School of Computer Science, University of Lincoln, United Kingdom. He worked in BJUT as a Lecturer (1996-1998) and an Associate Professor (1998-1999). He was a Senior Research Assistant at MEEM, City University of Hong Kong (1998-1999). He was an Alexander von Humboldt Research Fellow (2000, 2001) at the University of Kaiserslautern, Germany. Before joining the University of Lincoln as a Senior Lecturer (2007) and promoted to Reader (2010) and Professor (2012), he held research positions in the University of Cambridge (2006-2007), Newcastle University (2003-2006) and the University College London (UCL) (2002-2003) respectively. His research interests are mainly within the field of artificial intelligence, computer vision, robotics, brains, and neuroscience. He is particularly interested in biological visual neural systems and their applications in unmanned ground/aerial vehicles, interactive systems and robotics. He has published more than one hundred journal and conference papers in the above research areas. He is the founding director of Computational Intelligence Laboratory (CIL) in Lincoln and the deputy director of Lincoln Centre for Autonomous Systems (L-CAS). He has been a Guest Professor in XJTU (2014-2017). He is the coordinator for several EU FP7 and Horizon 2020 projects. Prof. Yue is a Member of IEEE, INNS and ISBE.

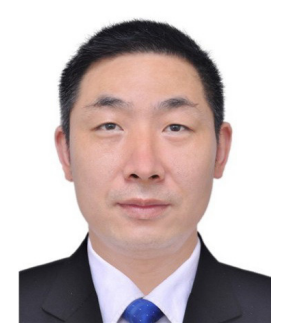

Zhuhong Zhang received the M.Sc. degree from Guizhou University, Guiyang, China, in 1998, and the Ph.D degree from College of Automation, Chongqing University, Chongqing, China, in 2004.

$\mathrm{He}$ is currently a Professor at the Guizhou University and an Associate Editor for Journal of Applied Soft Computing. His main areas of interests include uncertain programming, evolutionary algorithms, immune optimization, and signal simulation. He has also published more than 50 journal and conference papers in control theory, intelligent computing, project planning management and neural network. 